Science

Docs.

NBS MONOGRAPH 50

Bibliography on

Atomic Transition Probabilities

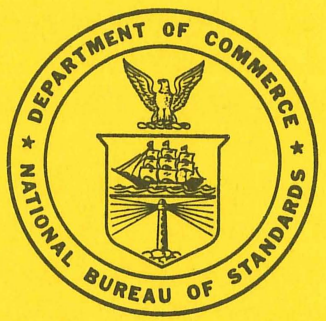

U.S. DEPARTMENT OF COMMERCE

NATIONAL BUREAU OF STANDARDS 


\section{THE NATIONAL BUREAU OF STANDARDS}

\section{Functions and Activities}

The functions of the National Bureau of Standards are set forth in the Act of Congress, March 3, 1901, as amended by Congress in Public Law 619, 1950. These include the development and maintenance of the national standards of measurement and the provision of means and methods for making measurements consistent with these standards; the determination of physical constants and properties of materials; the development of methods and instruments for testing materials, devices, and structures; advisory services to government agencies on scientific and technical problems; invention and development of devices to serve special needs of the Government; and the development of standard practices, codes, and specifications. The work includes basic and applied research, development, engineering, instrumentation, testing, evaluation, calibration services, and various consultation and information services. Research projects are also performed for other government agencies when the work relates to and supplements the basic program of the Bureau or when the Bureau's unique competence is required. The scope of activities is suggested by the listing of divisions and sections on the inside of the back cover.

\section{Publications}

The results of the Bureau's research are published either in the Bureau's own series of publications or in the journals of professional and scientific societies. The Bureau itself publishes three periodicals available from the Government Printing Office: The Journal of Research, published in four separate sections, presents complete scientific and technical papers; the Technical News Bulletin presents summary and preliminary reports on work in progress; and Basic Radio Propagation Predictions provides data for determining the best frequencies to use for radio communications throughout the world. There are also five series of nonperiodical publications: Monographs, Applied Mathematics Series, Handbooks, Miscellaneous Publications, and Technical Notes.

A complete listing of the Bureau's publications can be found in National Bureau of Standards Circular 460, Publications of the National Bureau of Standards, 1901 to June 1947 (\$1.25), and the Supplement to National Bureau of Standards Circular 460, July 1947 to June 1957 (\$1.50), and Miscellaneous Publication 240, July 1957 to June 1960 (Includes Titles of Papers Published in Outside Journals 1950 to 1959) (\$2.25); available from the Superintendent of Documents, Government Printing Office, Washington 25, D.C. 
UNITED STATES DEPARTMENT OF COMMERCE - Luther H. Hodges, Secretary NATIONAL BUREAU OF STANDARDS - A. V. Astin, Director

\section{Bibliography on}

\section{Atomic Transition Probabilities}

B. M. Glennon and W. L. Wiese

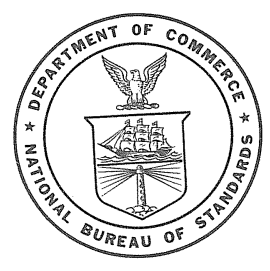

National Bureau of Standards Monograph 50 Issued August 1, 1962 



\section{Contents}

1.1. The NBS data center. . . . . . . . . . . . . . . . . . . . . .

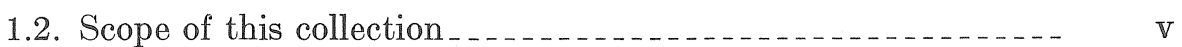

1.3. Availability of data_................................. VI

1.4. Arrangement of the bibliography _..... VII

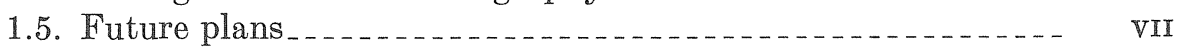

1.6. Table 1. Availability of atomic transition probabilities_... _ VIII

1.7. Table 2. Classification of articles_......................... IX

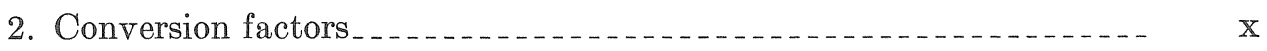

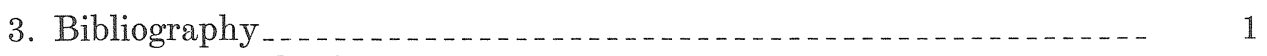

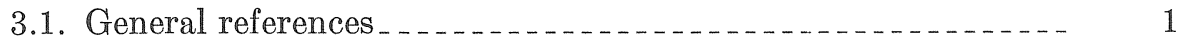

3.2. References on individual elements....................... 4

4. Partial list of abbreviations for journals _.................... 42 



\title{
Bibliography on Atomic Transition Probabilities ${ }^{1}$
}

\author{
B. M. Glennon and W. L. Wiese
}

\begin{abstract}
A bibliography on atomic transition probabilities is presented. The papers are arranged according to elements and stages of ionization, and the method employed and class of transitions are indicated behind each reference. Only articles on discrete transitions, both permitted and forbidden, are listed. Also included is a supplementary list of papers dealing with transition probabilities from a general point of view, a table showing the availability of numerical material on the individual atoms and ions, and a table of conversion factors.
\end{abstract}

\section{Introduction}

The numerical data on atomic transition probabilities are found widely scattered in the literature of the last 40 years, and the articles are often difficult to detect or quite inaccessible to the average user, as in the case of dissertations and technical reports. In view of the increasing need for these constants and the complexity of new calculations or experiments to determine them, it is imperative to assemble and make generally known all the material which is already available. The present collection is intended as a contribution to this end.

\subsection{The NBS Data Center}

In June 1960, a data center on atomic constants was established in the Atomic Physics Division of the National Bureau of Standards. The objectives of the center are to collect and catalog all relevant literature, to extract and analyze the numerical data, and to prepare and publish bibliographies and tables of "best" values. Work is in progress on collision cross sections and transition probabilities.

The collection of literature on transition probabilities was started by utilizing some recent survey articles. Branching out from these, all references and footnotes in the literature were scanned. Simultaneously, several abstracting journals were searched backwards through the year 1950 until a complete overlap with the available compilations was insured. Two card files ordered to authors and elements were established, and reprints of the articles were collected and cataloged. A large percentage of the articles are in languages other than English; translations are being made whenever the

1 This research is a part of project DEFENDER, sponsored by the Advanced Research Projects Agency, Department of Defense, through the
Office of Naval Research. language skills of the data center group do not suffice for a critical evaluation. The literature collection is now completely up-to-date, ${ }^{2}$ and the current literature including several abstracting journals is constantly being monitored.

\subsection{Scope of this Collection}

Since the public announcement of the data center in the summer of 1961, a large number of requests for bibliographies on individual elements have been received. It seems appropriate at this time to prepare a general bibliography, especially in view of the fact that the last two compilations by Kolesnikov and Leskov (Uspekhi Fiz. Nauk 65, 3, 1958) and by van Regemorter (J. phys. radium $\mathbf{2 0}, 907,1959$ ) are now already 3 years old, and thus do not contain a number of important papers published since then. The increased activity in this field in the last few years is partly reflected in the greater number of papers accumulated in this collection, namely well over 600, as against 370 by Kolesnikov and Leskov (which also includes molecular transitions) and about 250 by van Regemorter. Another reason for the increased size of this collection is that emphasis was laid on a complete presentation of the literature without regard to the quality of the article or to the application. Therefore, all of the early work was included. In many instances this is superseded by more refined determinations, but in cases where nothing else is available this older material is still very valuable for applications where a rough estimate will do. For the first 10 elements, for which a critical evaluation of the numerical data is under way, all work superseded by more refined determinations or considered not reliable is marked by an asterisk.

\footnotetext{
2 A few requests for theses, etc., are still outstanding.
} 
The bibliography is restricted to atomic and ionic transition probabilities of discrete transitions, including permitted (electric dipole) as well as forbidden (magnetic dipole, electric quadrupole) lines. The " $f$-values" for bound-free and free-free transitions are collected by another group of the data center under absorption cross sections, and the literature may be requested there (Atomic Constants Data Center-Collision Cross Sections).

\subsection{Availlability of Atomic Transition Prob- abilities}

The availability of transition probabilities for the individual elements is shown in column 4 of table 1. It is seen that for some of the 92 natural elements no material is available. For many other elements data exist only for the neutral atom and the first stage of ionization. Furthermore, the number of transitions treated is often quite small, and sometimes only the $f$-values for the resonance line or for forbidden lines are available. The latter case is indicated in the table by a subscript " $f$ " following the stage of ionization.

Fortunately, the available literature is supplemented by a few general treatments:

\section{a. The Coulomb Approximation by D.R. Bates and A. Damgaard}

Bates and Damgard (Phil. Trans. Roy. Soc. London, Ser. A, 24.2, 101, 1949) have shown there that the main contribution to the transition integral comes from a region in which the departure of the potential of an atom or ion from its asymptotic form is so small that it may.be replaced by the latter. This enables them to derive a general analytical expression for the transition integral and to compile tables (for $s-p, p-d, d-f$ transitions). From these the transition probabilities for any atom or ion can immediately be calculated, provided that the lower and upper energy levels of the transitions are known (for certain other restrictions see their article). Comparison of this method with other theoretical and experimental results shows that for simple atoms, i.e., those with one electron outside closed shells, the agreement is very good (with deviations usually smaller than 10 percent). Also, for many of the more complex atomic systems, especially in lighter elements, fair agreement is obtained.

Following the development of the Coulomb approximation a number of publications, especially in the astrophysical literature, contained numerical evaluations of a few transition probabilities using this method. None of these are included in this collection, except a few articles that contain a large amount of calculated values (more than 10 multiplets per stage of ionization). It is probably less time consuming and safer to evaluate the $f$-values rather than to look up articles in the hope of finding just the particular transitions. Secondly, very few of the authors state the applicability of the Coulomb approximation for the individual lines, i.e., the degree of interference in the transition integral, etc., providing thereby incomplete information anyway.

\section{b. The Screening Approximation by D. Layzer and C. M. Varsarsky}

This theoretical approach was recently developed by D. Layzer (Ann. Phys. 8, 271, 1959) and applied to a large number of transitions by $\mathrm{C}$. M. Varsavsky (Thesis, Harvard, 1958; and Astrophys. J. Suppl. Ser. 6, No. 53, 75-108, 1961). The method makes use of wave functions expanded explicitly in powers of the nuclear charge; consequently it is especially suitable for the simultaneous treatment of all elements in an isoelectronic sequence. In its simplest form, the approximation is based on screened hydrogenic functions. The results do not always agree well with values obtained from other methods; however, further refinements of the theory are planned. All elements treated in Varsavsky's second, more comprehensive paper (1961) are listed in column 6 of table 1 . In this article all transition probabilities are for lines in the vacuum ultraviolet.

\section{c. "Experimental Transition Probabilities for Spectral Lines of Seventy Elements" by C. H. Corliss and W. R. Bozman (National Bureau of Standards Mono- graph 53, 1962)}

Recently Meggers, Corliss, and Scribner have published tables of spectral line intensities for 39,000 lines of 70 elements in the wavelength range from 2000-9000A. ${ }^{3}$ They determined the intensities photographically from the spectra emitted by a free-burning $d-c$ arc with copper electrodes, into which 0.1 atomic percent of each element was mixed. In this way approximately uniform conditions for all elements were obtained. The temperature of the arc was determined by comparing the measured intensities with known relative $f$-values. This could be done for 20 elements and good agreement was obtained, indicating the general consistency of the data. The derived $f$-values are being published in an NBS Monograph, "Experimental Transition Probabilities for Spectral Lines of Seventy Elements," by C. H. Corliss and W. R. Bozman. Although errors in the absolute values as large as factors of 2 do not seem to be unreasonable, this work should be valuable for all applications where high accuracy is not required. The availability of this material is presented in table 1 , column 5 .

3 "Tables of Spectral Line Intensities," by W. F. Meggers, C. H, Corliss, and B. F. Scribner, National Bureau of Standards Monograph 32, October 1961 . 


\section{d. Two Comprehensive Papers on Forbidden Lines}

Among the many papers on calculations of $f$-values for forbidden lines are two that treat a large number of atoms and ions in which certain configurations occur. These comprehensive articles are not included in the element list in order to keep the size of this compilation compact. Instead, they are listed in table 1, column 7 (A. M. Naqvi, Thesis Harvard, 1951) and column 8 (S. Pasternack, Astrophys. J. פ2, 129, 1940).

\subsection{Arrangement of the Bibliography}

The bibliography is divided into two parts. The first part (section 3.1) contains all papers which deal with the subject of atomic transition probabilities from a general point of view. The articles are arranged according to the following subjects:

A. Tables of numerical values

B. Previous literature compilations

C. Review articles

D. Fundamental relationships and basic concepts

E. Detailed descriptions of experimental or theoretical methods

F. General comments

G. Environmental influences on transition probabilities.

Short explanatory remarks are given whenever it was felt necessary. In part 2 all literature containing numerical values on the individual elements is compiled except the articles listed in table 1 . It is arranged according to elements, these being listed in alphabetical order by their chemical symbol. Within the elements, the degrees of ionization serve as subdivisions, and the papers are listed in chronological order. Thus, for looking up $f$-value literature one should preferably start from the end of the list and work backward when the more recent work is exhausted.

For the abbreviations of the journals the designations in the "List of Periodicals" of Chemical Abstracts (1956 and Supplements) have been adopted and should be consulted when in doubt. For convenience, a list of abbreviations for the lesser known journals encountered here is attached at the end of this collection. Particular attention should be given to the apparent differences in the designation of some Russian journals. For instance, articles from the "Zhurnal Eksperimental'noi i Teoreticheskoi Fiziki" available only in the Russian language are listed as "Zhur. Eksptl. i Teoret. Fiz." If they have been or are being translated privately for the data center, the word "translated" is added in parentheses (transl.). Since 1958 the more important
Russian literature has been translated by the American Institute of Physics and other institutions. The translated versions are quoted. In this example the abbreviation is "Soviet Phys.JETP."

A number of Russian workers have published their articles in Russian journals and also translated in German journals (e.g., Prokof'ev, V. K., Z. Physik 5\%, 387 (1929) and Prokof'ev, V. K., Zhur. Eksptl. i Teoret. Fiz.1, 123 (1931) for Na I). In these cases a remark indicating this is added in parentheses behind the designation. The same is done when a dissertation, besides being published in full, is put into a journal in a condensed form or when, in addition to a paper, talks on the same subject are recorded in conference proceedings.

Under the column "classification" each article is characterized by one or more code words which indicate the method being used and the class of transitions being investigated, i.e., permittedelectric dipole-lines (nothing added) or forbidden-electric quadrupole, magnetic dipole-lines ("forb." added). The word relative ("rel.") appears in all cases where only relative transition probabilities are given. Explanations for the code words are given in table 2.

\subsection{Future Plans}

It is intended to publish additions or revised editions of this bibliography whenever significant amounts of new literature are accumulated. The ultimate goal of the data center is the publication of tables containing "best" numerical values of transition probabilities. Work on this project is in progress, and the material on the lightest 10 elements should be evaluated by the end of this year. However, the preparation of numerical data for all elements must be considered as a longrange program because of the enormous amount of work involved. It is our hope, that in the meantime the present bibliography may be of assistance to all interested scientists.

We express our sincere thanks to Dr. R. Hefferlin, who assisted effectively in getting this collection started, and who keeps a very keen interest in the progress of the center, and to Mary Lake and Berry Cobb, who did most of the tedious initial literature search. Melvin W. Smith participated in the final setup of this bibliography and offered many helpful suggestions.

We further thank all those who have generously furnished us with reprints, copies of dissertations, and technical reports. Our special thanks are due to Prof. Minnaert, Utrecht, and Prof. W. LochteHoltgreven, Kiel. 


\subsection{Table 1.-Availability of Atomic Transition Probabilities}

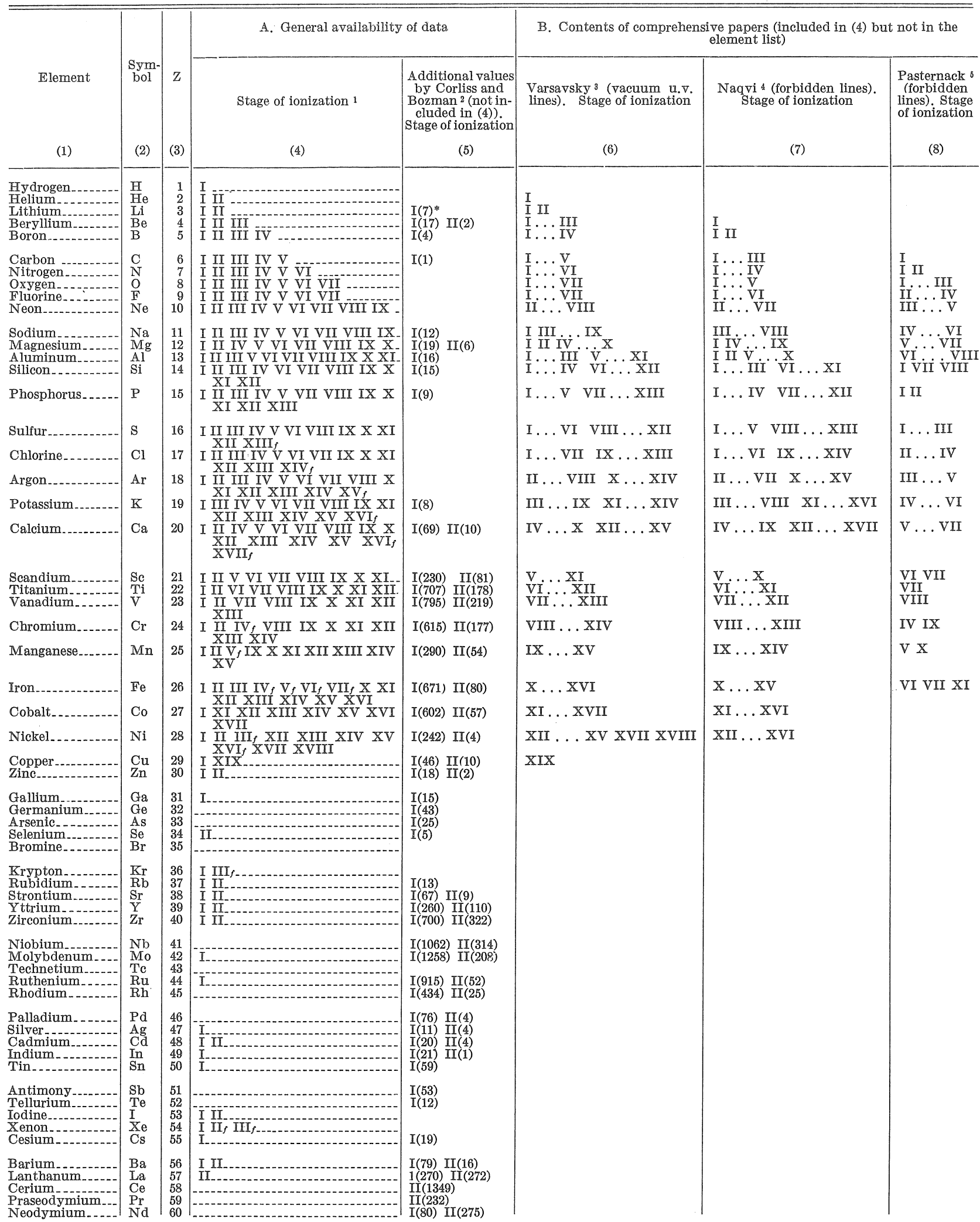




\subsection{Table 1.-Availability of Atomic Transition Probabilities-Continued}

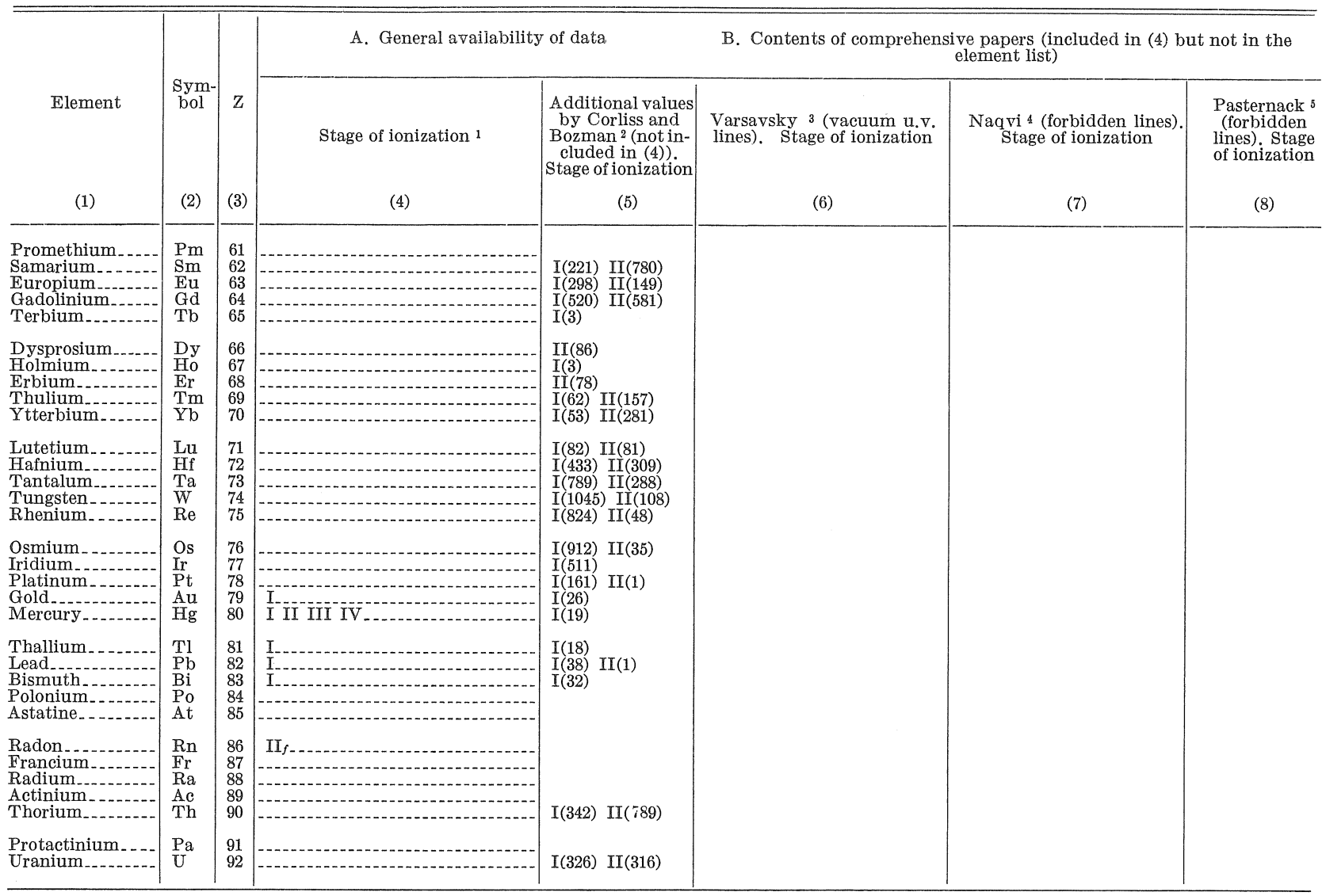

1 The stage of ionization is indicated by a Roman numeral ( $I=$ neutral atom, II = first ion, etc.). A subscript " $f$ " after the ion indicates that only data on forbidden lines are available. Hydrogen-like ions are generally not included in the bibliography. For relations to hydrogen see section 2 (Conversion Factors).

2 Corliss, C. H., a nd Bozman, W. R., National Bureau of Standards Monograph 53 (1962): "Experimental Transition Probabilities for Spectral Lines of Seventy Elements." (See section 1.3 for discussion.)

\subsection{Table 2.-Classification of Articles}

Except where "rel." (relative) appears, all determinations in the papers listed give absolute values.
A. Theoretical methods:
1. SCF-self-consistent field calculations
2. CA-Coulomb approximation
3. Quant.-other quantum mechanical calculations
4. Quant. forb.-quantum mechanical calculations for forbidden lines
5. Estim.-estimations from sum rules, etc.

B. Experimental methods:
1. Canal-canal ray experiments

3 Varsavsky, C. M., Astrophys. J. Suppl. Ser. 6, No. 53, 75 (1961). ${ }^{4}$ Naqvi, A. M., Thesis Harvard (1951)

${ }_{5}$ Pasternack, S., Astrophys. J. 92, 129 (1940); Publ. Astron. Soc. Pacific $\mathbf{5 1}, 160$ (1939). (The latter is superseded by the former.)

*The Arabic number indicates the number of lines measured.
2. Emiss.-measurement in emission (arc, furnace, discharge tube, etc.)

3. Absorpt.-measurement in absorption (King furnace, absorption tube, etc.)

4. Life-direct lifetime measurements

5. Hook-anomalous dispersion measurements

6. Misc.-miscellaneous experimental methods (magneto-rotation, polarization, magnetic resonance, astrophysical measurements, etc.)

C. Miscellaneous:

1. Comm.-comments, additions, or corrections to other articles, methods, etc.

2. Incompl. - article not yet classified (because translation is needed, incomplete information available, etc.)

3. Compil.-compilations 


\section{Conversion Factors}

The numerical factors relating the quantities $A_{k i}, f_{i k}$, and $S$ are given in table 3 , where:

$A_{k i}$ is the transition probability for spontaneous emission $\left(\mathrm{sec}^{-1}\right)$.

$f_{i k}$ is the absorption oscillator strength (dimensionless).

$S$ is the line strength in atomic units, which are:

for electric dipole transitions (alloweddenoted by $\mathbb{E}_{d}$ ):

$$
a_{0}^{2} e^{2}=6.459_{4} \times 10^{-36} \mathrm{~cm}^{2} \mathrm{esu}^{2} ;
$$

for electric quadrupole transitions (forbidden-denoted by $E_{q}$ ):

$$
a_{0}^{4} e^{2}=1.808_{8} \times 10^{-52} \mathrm{~cm}^{4} \mathrm{esu}^{2} ;
$$

for magnetic dipole transitions (forbidden-denoted by $M_{d}$ ):

$$
e^{2} h^{2} / 16 \pi^{2} m_{e}^{2} c^{2}=8.599 \times 10^{-41} \mathrm{erg}^{2} \text { gauss }^{-2} \text {. }
$$

The wavelength $\lambda$ is given in Angstrom units. $g$ is the statistical weight.

The factor in each square converts by multiplication the quantity above it into the one at its left. The upper energy level of a transition is denoted by the subscript $k$, the lower by $i$. The initial state is written first, i.e., $i$ first means absorption.

Besides the quantities introduced above, the following are also in use and are related to them by:

1. Transition probability of absorption $B_{i k}$

$$
B_{i k}=6.01 \lambda^{3} \frac{g_{k}}{g_{i}} A_{k i}
$$

2. Transition probability of induced emission $B_{k i}$

$$
B_{k i}=6.01 \lambda^{3} A_{k i}
$$

3. Emission oscillator strength $f_{k i}$

$$
f_{k i}=-\frac{g_{i}}{g_{k}} f_{i k} \text {. }
$$

\begin{tabular}{|c|c|c|c|}
\hline & $A_{k i}$ & $f_{i k}$ & $S$ \\
\hline \multirow{3}{*}{$A_{k i}$} & \multirow{3}{*}{1} & \multirow{3}{*}{$\frac{6.670_{2} \times 10^{15}}{\lambda^{2}} \frac{g_{i}}{g_{k}}$} & $\begin{array}{l}E_{d} \\
\frac{2.026_{1} \times 10^{18}}{g_{k} \lambda^{3}}\end{array}$ \\
\hline & & & $\begin{array}{l}E_{q} \\
\frac{1.679_{3} \times 10^{18}}{g_{k} \lambda^{5}}\end{array}$ \\
\hline & & & $\begin{array}{l}M_{d} \\
\frac{2.697_{2} \times 10^{13}}{g_{k} \lambda^{3}}\end{array}$ \\
\hline \multirow{3}{*}{$f_{i k}$} & \multirow{3}{*}{$1.4992 \times 10^{-16} \lambda^{2} \frac{g_{k}}{g_{i}}$} & \multirow{3}{*}{1} & $\frac{303.75}{g_{i} \lambda}$ \\
\hline & & & $\begin{array}{ll}E_{q} & \frac{251.8}{g_{i} \lambda^{3}} \\
\end{array}$ \\
\hline & & & $\begin{array}{l}M_{d} \\
\frac{4.043_{\theta} \times 10^{-3}}{g_{i} \lambda}\end{array}$ \\
\hline \multirow{3}{*}{$S$} & $E_{4.935_{6} \times 10^{-19} g_{k} \lambda^{3}}$ & $E_{d} \quad 3.292_{1} \times 10^{-3} g_{i} \lambda$ & \multirow{3}{*}{1} \\
\hline & $E_{q .953 \times 10^{-19} g_{k} \lambda^{j}}$ & $E_{q}{ }_{3.971 \times 10^{-3} g_{i} \lambda^{3}}$ & \\
\hline & $M_{d .707_{6} \times 10^{-14} g_{k} \lambda^{3}}$ & $\begin{array}{ll}M_{d} & \\
& 247.3_{0} g_{i} \lambda\end{array}$ & \\
\hline
\end{tabular}

Finally, it should be noted that the line strength $S$ is symmetrical:

$$
S=S_{i k}=S_{k i}
$$

TABLP 3.-Conversion factors

Since hydrogen-like ions (with one electron and nuclear charge $Z$ ) have intensity quantities which are simply related to hydrogen, papers dealing with these ions are not included in the bibliography. For convenience, the relations are given below:

$$
f_{Z}=f_{H} \quad S_{Z}=Z^{-2} S_{H} \quad A_{Z}=Z^{4} A_{H} .
$$

References for Section 2

Allen, C. W., Astrophysical Quantities (The Athlone Press, London, 1955).

Aller, L. H., Astrophysics: The Atmosphere of the Sun and the Stars (Ronald Press, 1953).

Condon, E. U., and Shortley, G. H., Theory of Atomic Spectra (University Press, Cambridge, 1959).

Unsöld, A., Physik der Sternatmosphären, 2d ed. (SpringerVerlag, Berlin, 1955).

The numerical values for the natural constants were taken from: Cohen, E. R., Crowe, K. M., and Dumond, J. W. M., Fundamental Constants of Physics (Interscience Publishers, Inc., New York, 1957). 


\subsection{GENERAL REFERENCES}

\section{A. Tables of Numerical Values}

Allen, C. Wo, "Astrophysical Quantities", 51-78 (The Athlone Press, London, 1955).

Biermann, L., "Zahlenwerte und Funktionen", 260-275 (ed. Landolt-Börnstein, Springer-Verlag, Berlin, 1950).

Filippov, A. N., Trudy Gosudarst. Opt. Inst. Leningrad 8, 1-118 (1932) (on the alkalis, Zn I, Cd I and

$\mathrm{Hg} \mathrm{1).}$

Goldberg, L., Mioller, E. A. E Aller, L. H., Astrophys. J. Suppl. Ser. 5, \#45, 1-137 (1960).

Korff, S. A. E Breit, G., Rev. Mod. Phys. 4, 471-503 (1932).

Mitchell, A. C. G. E Zemansky, M. W., "Resonance Radiation and Excited Atoms", ch. 3, $92=153$ (University Press, Cambridge, 1954).

Pearce, W., "Symposium on Optical Spectrometric Measurements of High Temperature", 142-169 (ed. Dickerman, P. Jo, University Press, Chicago, 1960).

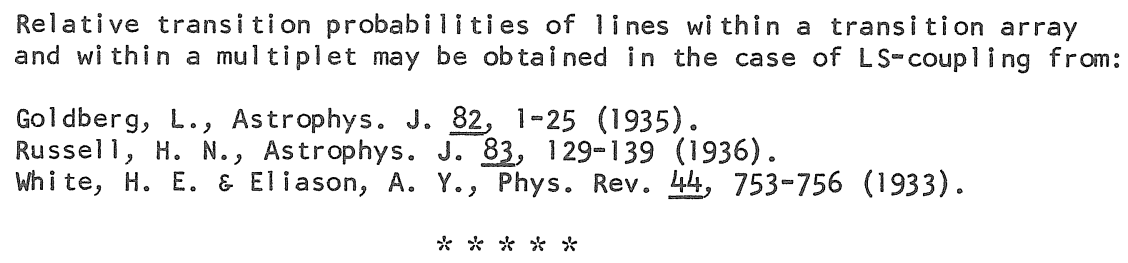

\section{B. Previous Literature Compilations*}

Aller, L. H., "Handbook of Physics", ch. 3, sec. 7, 48-57 (ed. Condon E Odishaw, McGraw-Hill Book Co., New York, 1958).

Claas, W. Jo, Recherches Astronomiques de l'observatoire d'Utrecht, 12, pt. 1, ch. 3, 21-52 (1951). Garstang, R. H., "Vistas in Astronomy", 1, 268-276 (ed. Beer, A., Pergamon Press, New York 1955). Hunger, K., Z. Astrophys. 36, 42-97 (1955). Kolesnikov, V. N. E Leskov, L. V., Uspekhi Fiz. Nauk 65, 3-38 (1958). van Regemorter, H., J. phys. radium 20, 907-914 (1959). Unsöld, A., "Physik der Sternatmosphären", 2nd ed., 269 (Springer-Verlag, Berlin, 1955).

$$
* * * * *
$$

\section{Review Articles}

Aller, L. H., "Astrophysics: The Atmosphere of the Sun and the Stars", 106-156 (Ronald Press Co., New York, 1953).

Aller, L. Ho, "Gaseous Nebulae", 164 (John Wiley \& Sons, Inc., New York, 1956).

Aller, L. H., "Handbook of Physics", ch.3, sec. 7, 48-57 (ed. Condon \& Odishaw, McGraw=Hill Book Co., New York, 1958).

Aller, L. H., "The Abundance of the Elements", 87-96 (Interscience Publisher, Inc., New York, 1961). Bowen, I. S., Rev. Mod. Phys. 8, 55-81 (1936) (on forbidden lines).

Filippov, A. N., Trudy Gosudarst. Opt. Inst. Leningrad 8, 1-118 (1932) (on the alkalis, Zn I, Cd I and $\mathrm{Hg} \mathrm{1)}$.

Frisch, S. E., J. Opt. Soc. Am. 50, 400-404 (1960) (current Russian work). [Translation of Uspekhi Fiz. Nauk 68, 3-12 (1959).] 
Garstang, R. H., "Vistas in Astronomy" 1, 268=276 (ed. Beer, A., Pergamon Press, New York, 1955). Goldberg, L., Mưller, E. A. E Aller, L. H., Astrophys. J. Suppl. Ser. 5, \#45, 1=137 (1960).

Jaffe, G., "Handbuch der Experimentalphysik" 19, 236-245 (ed. Wien-Harms, 1928).

Joos, G. E van Angerer, E., "Handbuch der Experimentalphysik" 21, 137-146 (ed. Wienm Harms, 1927).

King, R. B., Proceedings of the National Science Foundation Conference on Stellar Atmospheres, Indiana University, $41=43$ (1954).

Kolesnikov, V. N. E Leskov, L. V., Uspekhi Fiz. Nauk $65,3-38$ (1958).

Korff, S. A. E Breit, G., Rev. Mod. Phys. $4,471-503$ (1932).

Ladenburg, R., Naturwissenschaften 17, 296-299 (1929) (on anomalous dispersion experiments).

Ladenburg, R., Z. El ektrochem. 36, 631-640 (1930).

Ladenburg, R. E Reiche, F., Naturwissenschaften 27, $584-598$ (1923).

Layzer, E., Proceedings of the Tenth International Astrophysical Colloquium, Liège, Belgium (1960) (to be published) (on theoretical methods).

Mitchel1, A. C. G. E Zemansky, M. W., "Resonance Radiation and Excited Atoms", ch. 3, 92-153 (University Press, Cambridge, 1934).

Mrozowski, So, Rev. Mod. Phys. 16, 153-174 (1944) (experiments on forbidden lines).

Naqvi, A. M., Geophysics Corporation of America Technical Report 61-21-A; ASTIA Document 263459 (1961). van Regemorter, $H_{0}$, J. phys. radium $20,907=914$ (1959) (on experimental methods).

Rubinowicz, A. E Blaton, J., Ergeb. exakt. Naturw. 11, 176-217 (1932) (on forbidden 1ines).

Transactions of the International Astronomical Union, vols. VII (1950), VIII (1952), IX (1955), X (1958) and XI (1961).

Unso̊ld, A., "Physik der Sternatmosphåren', 2nd ed., 269 (Springer-Verlag, Berlin, 1955).

$$
* * * * *
$$

\section{Fundamental Relationships and Basic Concepts*}

Condon, E. U. E Shortley, G. H., "Theory of Atomic Spectrall (University Press, Cambridge, 1959) (introduction of line strengths).

Einstein, A., Verhandl. deut, physik. Ges.18, 318-323 (1916) (introduction of transition probabilities). Einstein, A., Physik. Z. 18, 121-128 (1917) (introduction of transition probabilities).

Kirkwood, J. G., Physik. Z. $33,521-525$ (1932) (sum rule).

Kuhn, W., Z. Physik 33, 408-412 (1925) (sum rule).

Ladenburg, R., Z. Physik 4, 451-468 (1921) (relation between frvalue and transition probability).

Reiche, F. E Thomas, Wo, Z. Physik 34, 510-525 (1925) (sum rule).

Wigner, E., Physik. Z. $32,450-453$ (1931) (sum rule).

$$
* * * * *
$$

\section{E. Detailed Descriptions of Experimental or Theoretical Methods}

Bates, D. R. E Damgaard, A., Phil. Trans. Roy. Soc. London. Ser. A. 242, 101-122 (1949) (Coulomb approximation).

Bethe, H. A. E Salpeter, E. E., "Quantum Mechanics of One and Two Electron Atoms" (Academic Press, New York, 1957).

Biberman, L. M., Optika i Spektroskopiya 2, $397=399$ (1957).

Condon, E. U. E Shortley, G. H., "Theory of Atomic Spectrall (University Press, Cambridge, 1959).

Garstang, R. H., Proc. Cambridge Phil. Soc. 53, 214-221 (1957) (forbidden lines).

Garstang, R. H., Proc. Cambridge Phil. Soc. 54, 383-390 (1958) (forbidden lines).

Hartree, D. R., "The Calculation of Atomic Structures" (John Wiley \& Sons, Inc., New York, 1957).

Kagan, Yu. M. E Koritskii, Ya. P., Optics and Spectroscopy (U.S.S.R.) 11, 166-168 (196i) (1ifetime).

Mitchell, A. C. G. E Zemansky, M. W., "Resonance Radiation and Excited Atoms", ch. 3, 92-153 (University Press, Cambridge, 1954) (experimental).

Penner, S. S., "Fundamental Data Obtained from Shock-Tube Experiments", ch. 7 (ed. Ferri, A., Pergamon Press, New York, 1961).

Prokof"ev, V. Ko, Trudy Gosudarst. Opt. Inst. Leningrad No. 25, 1-31 (1924) (anomalous dispersion). Rozhestvenskii, D. So, "Raboty po Anomal noi Dispersi i v Parax Metallov" (Izdatel'stvo Akademi $i$ Nauk S.S.S.R., 1951).

Rozhestvenskii, D. S. E Penkin, N. P., J. Phys. (U.S.S.R.) 5, 319-337 (1941) (anomalous dispersion). slater, J. C., Phys. Rev. 25, 783-790 (1925) (absorption).

slater, J. Co, "Quantum Theory of Atomic Structure", vol. 2 (McGraw-Hill Book Co., New York, 1960). Soleillet, P., Compt. rend. 239, 698-700 (1954) (1 ifetime).

Zastavenko, L. G. E Khrustalev, O. A., Optics and Spectroscopy (U.S.S.R.) 11, $241-242$ (1961) (1ifetime).

$$
* * * * *
$$

*See also footnote for section $F$. 
Allen, C. W. E Asaad, A. S., Monthiy Notices Roy. Astron. Soc. 115, 571-574 (1955) (on f-values for elements of the iron group).

Fock, V., Z. Physik 89, 744-749 (1934) (deviations in fosum rules).

Goldberg, L., Astrophys. J. 84, 11-13 (1936) (normalization factors for obtaining absolute line strengths).

Green, L. C.; Weber, N. E. E Krawitz, E., Astrophys. J. 113, 690-696 (1951) (use of observed and calculated energy values).

Gruzdev, P.F. E Startsev, G. P., Optics and Spectroscopy (U.S.S.R.) 8, 461-462 (1960) (criteria for LScoupling).

Hefferlin, R., J. Tenn. Acad. Sci. 36, 76-80 (1961) (semi-empirical investigations in isoelectronic sequences).

Horie, H., J. Phys. Soc. Japan Z, 58-61 (1952) (on line strengths).

Ladenburg, R., Naturwissenschaften 14, 1208-1213 (1926) (on the dispersion formula).

Menzel, D. H. E Goldberg, L., Astrophys. J. 84, 1=10 (1936) (multiplet strengths for transitions involving equivalent electrons).

Penner, S. S., J. Opt. Soc. Am. 43, 218-219 (1953) (use of relative intensity measurements).

Rohrlich, F., Astrophys. J. 129, 441-448 (1959) (on relative multiplet strengths).

Rohrlich, F., Astrophys. J. 129, 449-455 (1959) (on f-sum rules).

Shortley, G. H., Phys. Rev. $\frac{47}{47}$ 295-300 (1935) (1ine strengths in intermediate coupling).

Shortley, G. H., Phys. Rev. 47, 419 (1935) (comment on and addition to above).

Shortley, G. H., Phys. Rev. 57, 225-234 (1940) (general formulations for line strengths of forbidden transitions).

Shortley, G. H., Aller, L. H., Baker, J.G.E Menzel, D.H., Astrophys. J. 93, 178-184 (1941) (strengths of forbidden lines as a function of coupling).

Yamanouchi, T., Proc. Phys.-Math. Soc. Japan 23, 1059-1062 and 1063-1068 (1941) (on relative multiplet strengths).

$$
* * * * *
$$

\section{G. Environmental Influences on Transition Probabilities}

Galt, J.A. E Welsh, H. L., Can. J.Phys. 35, 98-113 and 114-121 (1957).

Michels, A., de Kluiver, H. E Castle, B., Physica 23, $1131-1142$ (1957).

Michels, A., de Kluiver, H. E Middelkoop, D., Physica 24, 543-544 (1958).

Mo̊glich, F。 E Rompe, R。, Z. Physik 120, 741-772 (1943)。

Strijland, J. C. E Nanassy, A. Jo, Physica 24, 935-936 (1958).

Trumpy, B., Z. Physik 40, 594-617 (1926).

Trumpy, B., Kgl. Norske Videnskab. Selskabs, Skrifter 1, 1-80 (1927).

$$
* * * * *
$$

*An extensive bibliography of articles dealing with radiative transitions from a general point of view is presented in, for example, "Quantum Theory of Atomic Structure", vol. 2 (McGraw-Hill Book Co., New York, 1960) by J. C. Slater. In this section only papers of practical interest are listed. 
$\mathrm{Ag} I$

Filippov, A. N. E Islamov, I. I.

Islamov, I. I.E Filippov,

A. N.

Terpstra, J.

Allen, C. W.

Allen, C. W. E Asaad, A. S.

Hinnov, E. E Kohn, H.

Terpstra, J. E Smit, J.A. Addink, N. W. H.

Voorhoeve, P. G.

Biermann, L.

Biermann, L. E Lübeck, K.

Parchevskii, G. F. E Penkin, N. P.

Penkin, N. P. E Parchevski i, G. F.

Nikonova, E. I. E Prokof'ev, V. K.

Allen, C. W.

Allen, C. W. E Asaad, A. S.

Ostrovskii, Yu. I.

Varsavsky, C. M.

Addink, N. W. H.

Goldberg, L., Mioiller, E. A. \& Aller, L. H.

Brehm, B., Demtröder, W. E Osberghaus, 0 .

Demtro̊der, W.

Eddy, J. A., House, L. L. E Zirin, $H$.

Eddy, J. A., House, L. L. E Zirin, $H$.

Hanus, W.

Korolev, F. A. E Kvaratskheli, Yu. K.

Demtro̊der, $W$.
Z. Physik 85, 409-410 (1933) (same as below).

Zhur. Eksptl.i Teoret. Fiz. 3, 524-525 (1933) (trans1.) (same as above).

Thesis Utrecht (1956). [See Terpstra, J. E Smit, J.A., Physica 24, 937-958 (1958) for results.]

Monthly Notices Roy. Astron. Soc. 117, 622-628 (1957).

Monthly Notices Roy. Astron. Soc. 117, 36-49 (1957) (see also above).

J. Opt. Soc. Am. 47, 156-i62 (1957).

Physica 24, 937-958 (1958).

spectrochim. Acta 15, 349-359 (1959).

$$
* * * * *
$$

\section{Al I}

Thesis Utrecht (1946).

Nachr. Akad. Wiss. Göttingen, Math.-physik. Kl. 116-118 (1946-1948) (see also below).

z. Astrophys. 25, 325-339 (1948).

Soviet Phys. - JETP I, 382-384 (1955) (see also below).

Zhur. Eksptl。 i Teoret. Fiz. 28, 766 (1955).

Optika i Spektroskopiya 1, 290-297 (1956)

(transl.).

Monthly Notices Roy. Astron. Soc. 117, 622= 628 (1957).

Monthly Notices Roy. Astron. Soc. 117, 36-39 (1957) (see also above).

Optika i Spektroskopiya 2, 673 (1957) (transl.). Thesis Harvard (1958).

Spectrochim. Acta 15, 349-359 (1959).

Astrophys. J. Suppl.Ser. 5, \#45, 1-137 (1960).

Z. Naturforsch. 16a, 843 (1961). [See also Demtrooder, W., Thesis Bonn (1961) and $Z$. Physik 166, 42-45 (1962).]

Thesis Bonn (1961). [See Z. Physik 166, 4245 (1962) for results.]

Astrophys. J. 133, 299-302 (1961) (see also below).

Astrophys. J. 134, 1028 (1961).

Bul1. acad.polon. sci. Classe 3, 8, 629-636 (1961).

Optics and Spectroscopy (U.S.S.R.) 10, 200-202 (1961).

z. Physik 166, 42-45 (1962).

\author{
hook, rel. \\ hook, rel. \\ emiss., rel. \\ comm. \\ emiss. \\ emiss. \\ emiss., rel. \\ emiss.
}

emiss., rel. SCF

SCF

hook, rel.

comm.

hook, rel.

comm.

emiss.

hook, rel.

quant.

emiss.

$\mathrm{CA}$

life

life

quant.

comm.

quant.

emiss., rel.

life 
Al \|\|

Gaustad, J.E. E Spitzer, Jr., L.

Wol fsohn, G.

Larsen, $T$.

Fursov, V. S., Oganov, M. N. E Striganov, A. R.

Drawin, H. W.

Petersen, R.

Knox, R. S.

olsen, H. N.

Doherty, L. R.

Gericke, W. E.

Garstang, R. H.

01 sen, H. N.

Gaustad, J.E. E Spitzer, Jr., L.

Osterbrock, D. E.

Naqvi, A. M. \& Talwar, S. P.

Edlén, B.

Osterbrock, D.E.

Edlén, B.

Addink, N. W. H.
Astrophys. J. 134, 771-776 (1961).

comm.

$$
* * * * *
$$

Ar 1

Z. Physik 85, 366-372 (1933).

Z. Physik 88, 389-394 (1934).

Doklady Akad. Nauk S.S.S.R. 101, 453-455 (1955)

(transl. $A E C=T R=2744$ ).

Z. Physik 146, 295-313 (1956).

Phys. \& Chem. Solids 1, 284 (1957).

Phys. Rev. 110, 375-381 (1958).

Bull. Am. Phys. Soc. (2) 4, 262 (1959).

Thesis Michigan (1961).

Z. Astrophys. 53, 68-79 (1961).

$$
\operatorname{Ar} \|
$$

Monthly Notices Roy. Astron. Soc. 114, 118-133 (1954).

Bull. Am. Phys. Soc. (2) 4, 262 (1959).

Astrophys. J. 134, 771-776 (1961).

\section{$\operatorname{Ar}\|\|$}

Astrophys. J. 114, 469-472 (1951).

quant. forb.

$$
\text { Ar IV }
$$

Monthly Notices Roy. Astron. Soc. 117, 463471 (1957).

Ar $X$

Z. Astrophys. 22, 30-64 (1942).

quant. forb.

Ar $X 1$

Astrophys. J. 114, 469-472 (1951).

quant. forb.

Ar XIV

Z. Astrophys. 22, 30-64 (1942).

quant. forb.

hook

hook \& estim.

emiss.

emiss.

quant., rel.

SCF

emiss.

emiss.

emiss.

quant., rel.

\& CA

emiss.

estim.

quant. forb.

$$
* * * * *
$$

Au I

Spectrochim. Acta 15, 349-359 (1959).

emiss. 
B I

Bolotin, A. B. E Yutsis, A. P.

Tsiunaitis, G. K. E Yutsis, A. P.

Veselov, M. G.

Bolotin, A. B. E Yutsis, A. P.

Veselov, M. G.

*Varsavsky, C.M.

Veselov, M. G.

Veselov, M. G.

Veselov, M. G.

Prokoflev, V. K.

Prokof"ev, V. K.

Chamalaun, F.J.

King, G. W. E van Vleck, J. $H$.

Rozhestvenskii, D. S. $\varepsilon$ Penkin, N. P.

Rozhestvenskii, D. S. E

Penkin, N. P.

Kruithof, A. M.

Kruithof, A. M.

Kruithof, A. M. E Smit, J. A.

Penkin, N. P.

Wessel, G.

Ostrovskii, Yu. 1., Penkin, N. P. E Shabanova, L. N.

Ostrovskii, Yu. I., Penkin, N. P. E Shabanova, L. N.
Zhur. Eksptl。i Teoret. Fiz. 24, 537=543 (1953) (transl.).

Soviet Phys。 = JETP $1,358=363$ (1955).

SCF

SCF

B \|

Zhur. Eksptl. i Teoret. Fiz. 19, 959-964 (1949) (ATS transl.).

Zhur. Eksptl。i Teoret. Fiz. 24, 537-543 (1953) (transl.).

Vestnik Leningrad Univ. Ser. Fiz. i Khim. No.8, $181-185$ (1953) (transl.).

Thesis Harvard (1958).

\section{$B\|\|$}

Zhur. Ekspti. i Teoret. Fiz. 19, 959-964 (1949) (ATS transl.).

\section{B IV}

Zhur. Eksptl. i Teoret. Fiz. 19, 959-964 (1949) (ATS transl.).

Vestnik Leningrad Univ. Ser. Fiz. i Khim, No. 8 , $181-185$ (1953) (transl.).

$$
* * * * *
$$

$\mathrm{Ba} I$

Z. Physik 50, 701-715 (1928) (same as below). Zhur. Eksptl. i Teoret. Fiz. I, 111-122 (1931) (transl.) (same as above).

Thesis Utrecht (1934).

Phys. Rev. 56, 464-465 (1939).

J. Phys. (U.S.S.R.) 5, 319-337 (1941).

Izvest. Akad. Nauk S.S.S.R. Ser. Fiz. 5, 97-101 (1941) (transl.).

Thesis Utrecht (1943) (see below for results). Physica 10, 493-501 (1943).

Physica I1, 129-143 (1944).

Izvest. Akad. Nauk S.S.S.R. Ser. Fiz. 11, 217= 220 (1947) (transi.).

Z. Physik 126, 440-449 (1949).

Bull. Acad. Sci. U.S.S.R. Phys. Ser. 22, 720724 (1958) (results same as below). [See al so Ostrovski i, Yu. I.EPenkin, N. P., Optics and Spectroscopy (U.S.S.R.) 11, 307= 309 (1961).]

Soviet Phys. - Doklady 3, 538=540 (1958)

(results same as above). [See also Ostrovskii, Yu. 1.EPenkin, N. P., Optics and Spectroscopy (U.S.S.R.) 11, 307-309 (1961).] quant.

SCF

quant.

quant.

quant.

quant.

quant.

hook, rel. hook, rel.

emiss., rel. \& quant., rel. quant. forb., rel. hook, rel. \& comm. hook, rel.

emiss., rel. emiss., rel. estim.

incomp l.

absorpt. hook

hook 
Addink, N. W. H. N.P.

Bucka, H. E Schussler, H. J. Ostrovski i, Yu. I. EPenkin, N.P.
Ostrovskii, Yu. I. EPenkin,

Spectrochim. Acta 15, 349-359 (1959).

Optics and Spectroscopy (U.S.S.R.) 2, $371-373$

(1960). [See also ibid. 11, $307-309$ (1961).]

Ann. Physik Z, 225-232(1961).

Optics and Spectroscopy (U.S.S.R.) 11, 307-309 (1961).

$\mathrm{Ba} \|$

Kerschbaum, $H$.

Mason, R. C.

Rozhestvenskii, D. S. E Penkin, N.P.

Kruithof, A. M.

Kruithof, A. M.

Kruithof, A. M. $\&$ Smit, J.A.

Reindl, H.P.

Nikonova, E. I. E Prokof'ev, V. K.

Boyarchuk, M. E. E Boyarchuk, A. A.

Belousova, 1. M. E Gurevich, D. B.

Ostrovskii, Yu. I. EPenkin, N. P.

Ostrovskii, Yu. I. E Penkin, N.P.

Bates, D. R. E Damgaard, A.

Biermann, L. E Trefftz, E. Veselov, M. G.

Bolotin, A. B. E Yutsis, A. P.

Veselov, M. G.

Greenstein, J.L. E Tandberg=Hanssen, $E$.

*Varsavsky, C. M.

Addink, N. W. H.

Veselov, M. G.

Greenstein, J.L. E Tandberg= Hanssen, E.

*Varsavsky, C. M.

Veselov, M. G.

Veselov, M. G.

Yutsis, A. P., Ushpalis, K. K., Kavetskis, $V_{0}$. . E Levinson, I. B.

$6356840-62-3$
Ann. Physik 83, 287-295 (1927).

Physica $5,777-783$ (1938).

J. Phys. (U.S.S.R.) 5, 319-337 (1941).

Thesis Utrecht (1943) (see below for results).

Physica 10, 493-501 (1943).

Physica 11, 129-143 (1944).

Thes is Utrecht (1946).

Optika i Spektroskopiya I, 290-297 (1956) (transl.).

Akademi ia Nauk S.S.S.R. Krymskaia astrofizecheskaia observatoriia. Izvesti ia 22, 234-256 (1960)

(transl.).

Optics and Spectroscopy (U.S.S.R.) 10, 206-207 (1961).

Optics and Spectroscopy (U.S.S.R.) 10, 3-6 (1961) (see al so below).

Optics and Spectroscopy (U.S.S.R.) 11, 307-309 (1961).

$* * * x *$

Be I

Phi 1. Trans. Roy. Soc. London. Ser. A. 242, 101122 (1949).

Z. Astrophys. 26, 213-239 (1949).

Zhur. Eksptl.i Teoret. Fiz. 19, 959-964 (1949) (ATS transl.).

Zhur. Eksptl. i Teoret. Fiz. 24, 537-543 (1953) (transl.).

Vestnik Leningrad Univ. Ser. Fiz. i Khim. No. 8 , $181=185$ (1953) (transi.).

Astrophys. J. 119, 113-119 (1954).

Thesis Harvard (1958).

Spectrochim. Acta 15, 349-359 (1959).

$\mathrm{Be} \|$

Zhur. Ekspt1。i Teoret. Fiz. 19, 959-964 (1949) (ATS transl.).

Astrophys. J. 119, 113-119 (1954).

quant.

comm。

Thes is Harvard (1958).

quant.

$\mathrm{Be}\|\|$

Zhur. Ekspt1. i Teoret. Fiz. 19, 959-964 (1949)

quant.

(ATS transi.).

Vestnik Leningrad Univ. Ser.Fiz. i Khim. No.8,

$181=185$ (1953) (transi.).

Optika i spektroskopiya 1, 601-605 (1956) (transl.).quant.

$$
* * * * * *
$$

Ba $\mid$ to $\mathrm{Be}\|\|$ 
Sobolev, N. N.

Allen, C. W.

Allen, C. W. E Asaad, A. S.
$B i$

Zhur. Eksptl. i Teoret. Fiz. 13, 131-136 (1943) (transi.).

Monthly Notices Roy. Astron. Soc. 117, 622-628 (1957).

Monthly Notices Roy. Astron. Soc. 117, 36-49

(1957) (see also above).

$* * * * *$

C I

* Stevenson, A. F.

*Condon, E. U.

Garstang, R. H.

Yamanouchi, T. E Horie, $H_{\text {. }}$

Maecker, H.

Yilmaz, H.

Bolotin, A. B., Levinson, 1. B. \& Levin, L. I.

Richter, J.

*Varsavsky, C. M.

Goldberg, L., Mưller, E. A. E Aller, L. H.

Doherty, L. R.

Foster, E. W.

Aller, L. H.

Biermann, L. E Lübeck, K.

Bolotin, A. B. E Yutsis, A. P.

Maecker, $H$.

*Varsavsky, C. M.

Aller, L. H. E Jugaku, J.

Gaustad, J.E. E Spitzer, Jr., L.

Aller, L. H.

Veselov, M. G.

Bolotin, A. B. E Yutsis, A. P.

Veselov, M. G.

Gaustad, J.E. E Spitzer, Jr., L.

Aller, L. H.

Veselov, M. G.
Astrophys. J. 79, 217-234 (1934).

Monthly Notices Roy. Astron. Soc. $111,115=124$ (1951).

J. Phys. Soc. Japan Z, 52-56 (1952).

Z. Physik 135, 13-22 (1953).

Phys. Rev. 100, 1148-1153 (1955).

Soviet Phys: - JETP 2, 391-395 (1956).

Z. Physik 151, 114-123 (1958).

Thesis Harvard (1958).

Astrophys. J. Supp 1. Ser. 2, \#45, 1-137 (1960).

Thesis Michigan (1961).

Proc. Phys. Soc. London A 79, 94-104 (1962).

\section{c $\|$}

Astrophys. J.97, 135-165 (1943).

Z. Astrophys. $25,325=339$ (1948).

Zhur. Ekspt1. i Teoret. Fiz. 24, 537-543 (1953)

(transl.).

Z. Physik 135, 13-22 (1953).

Thes is Harvard (1958).

Astrophys.J. Suppl.Ser.4, \#38, 109-156 (1959).

Astrophys. J. 134, $771-776$ (1961).

\section{C \|\|}

Astrophys. J. 27, 135-165 (1943).

Zhur. Ekspt1. i Teoret. Fiz. 19, 959-964 (1949)

(ATS transl.).

Zhur. Ekspt1. i Teoret. Fiz. 24, 537-543 (1953)

(transl。).

Vestnik Leningrad Univ. Ser. Fiz. i Khim. No. 8, 181-185 (1953) (trans1.).

Astrophys. J. 134, $771-776$ (1961).

\section{IV}

Astrophys. J.97, 135-165 (1943).

zhur. Eksptl. i Teoret. Fiz. 19, 959-964 (1949)

(ATS transi.).
Proc. Roy. Soc. London A 137, 298-325 (1932). emiss., rel.

comm.

emiss.

quant. forb quant. forb. quant. forb.

quant. forb. emiss. quant. forb. quant.

emiss. quant. CA

emiss. emiss.

quant.

SCF

SCF

emiss. quant. $C A$ comm. E estim.

quant. quant.

SCF

quant.

estim.

quant. quant. 
C V

Veselov, M. G.

Veselov, M. G.

Frerichs, R.

Kerschbaum, $H$.

Russell, H. N.

Prokof'ev, V. K.

Menzel, D. H.

Prokof'ev, V.K.

Filippov, A. E Kremenevsky, N. Chamalaun, F.J.

Steinhåuser, A.

Steinhåuser, A.

Katz, E. E Ornstein, L. S.

Hartree, D. R. E Hartree, W.

King, G. W. E van Vleck, J.H.

Schuttevaer, J.W.

Schuttevaer, J.W., de Bont,

M. J. E van den Broek, Th. H.

Bates, D. R. E Damgaard, A.

Trefftz, E.

Olsen, K. H., Routly, P. M. E King, R. B.

$0 l$ sen, K. H.

Allen, C.W.

Vainshtein, L. A.

Ostrovskii, Yu. 1., Penkin, N. P. E Shabanova, L. N.

Ostrovskii, Yu. 1., Penkin, N. P. E Shabanova, L. N.

Varsavsky, C. M.

Addink, N. W. H.

olsen, K. H., Routly, P. M. E King, R. B.

Boyarchuk, M. E. E Boyarchuk, A. A.

Goldberg, L., Můller, E. A. \& Aller, L. H.

Ostrovski i, Yu. I. E Penkin, N.P.

ostrovskii, Yu. I. EPenkin, N P.
Zhur. Eksptl. i Teoret. Fiz. 19, 959-964 (1949) (ATS transi.).

Vestnik Leningrad Univ. Ser. Fiz. i Khim. No.8, $181-185$ (1953) (transl.).

$\dot{*} * * * \dot{x}$

Ca I

Z. Physik 31, 305-310 (1925).

Ann. Physik 83, 287-295 (1927).

Astrophys. J. 78, 239-297 (1927).

Z. Physik 50,701-715 (1928). [Same as Zhur.

Ekspt1. i Teoret. Fiz. 1, 111=122 (1931).]

Publ. Lick Observatory 17, 232-238 (1930).

Zhur. Eksptl. i Teoret. Fiz. 1, 111-122 (1931)

(transl.). [Same as Z. Physik 50, 701-715 (1928).]

Physik. Z. Sowjetunion 1, 299-301 (1932).

Thesis Utrecht (1934).

Z. Physik 25, 669-686 (1935) (see also below).

Z. Physik 99, 300 (1936).

Physica $4,757-760$ (1937).

Proc. Roy. Soc. London A 164, 167-191 (1938).

Phys. Rev. 56, 464-465 (1939).

Thesis Utrecht (1942) (see below for results).

Physica 10, 544-552 (1943).

Phil. Trans. Roy. Soc. London. Ser. A. 242, 101= 122 (1949).

Z. Astrophys. 29, 287-303 (1951).

Proceedings of the National Science Foundation Conference on Stellar Atmospheres, Indiana Univ., p. 46 (1954).

Thesis Cal ifornia Institute of Technology (1956). [See 0lsen, K. H., et al., Astrophys. J. 130, 688-692 (1959) for results.]

Monthly Notices Roy. Astron. Soc. 117, 622628 (1957).

Optika i Spektroskopiya 3, 313-321 (1957) (transl.).

Bull. Acad. Sci. U.S.S.R. Phys. Ser. 22, 720= 724 (1958) (results same as below). [See also Ostrovskii, Yu. I. E Penkin, N. P., Optics and Spectroscopy (U.S.S.R.) 11, 307= 309 (1961).]

Soviet Phys. - Doklady 3, 538-540 (1958) (results same as above). [See also Ostrovskii, Yu. 1.E Penkin, N. P., Optics and Spectroscopy (U.S.S.R.) $11,307-309$ (1961).]

Thesis Harvard (1958).

Spectrochim. Acta 15, 349-359 (1959).

Astrophys. J. 130, 688-692 (1959).

Akademi ia Nauk S.S.S.R. Krymskaia astrofizecheskaia observatoriia. Izvestila 22, 234-256 (1960) (transl.).

Astrophys. J. Suppl.Ser. 5, \#45, 1=137 (1960).

Optics and Spectroscopy (U.S.S.R.) 10, 219-222 (1961) (see also below).

optics and Spectroscopy (U.S.S.R.) 11, 307-309 (1961). quant.

quant.

emiss., rel.

canal

estim.

hook, rel.

comm. E estim.

hook, rel.

hook, rel.

emiss., rel.

E quant., rel.

misc.

comm.

emiss., rel.

SCF

quant. forb., rel.

emiss., rel.

emiss., rel.

SCF

SCF

absorpt., rel.

absorpt.

emiss. E CA

incompl.

hook

hook

quant.

emiss.

absorpt.

comm 。

CA

hook, rel.

hook \& comm.

C V to Ca I 
Milne, M. E.

Russel1, H. N.

Zwaan, A.

Gerasimovic, B.P.E Struve, 0 .

Zwaan, A.

Zwaan, A.

Menzel, D. H.

Hartree, D. R. E Hartree, W.

Bates, D. R. E Damgaard, A.

Green, L. C. E Weber, N. E.

Green, L. C., Weber, N.E. E Krawitz, E.

Osterbrock, D. E.

Seaton, M. J.

Green, L. C., Weber, N. E. E Krawitz, E.

Treffiz, E. E Biermann, L.

Nikitin, A. A.

Nikitin, A. A. E Gordienko, G. V.

Nikonova, E. I. E Prokof'ev, V. K.

Bersuker, I. B.

Dvornikova, I. U. E Nagibina, I. M.

Nagibina, I. M.

Nagibina, I. M.

Varsavsky, C. M.

Houziaux, L. E Sadoine, M. P.

Korolev, F. A. E Kvaratskhel i, Yu. K.

Ostrovskii, Yu. I. EPenkin, N.P.

Ostrovskii, Yu. 1.EPenkin, N. P.

Douglas, A. S. E Garstang, R. H.

Osterbrock, D. E.

Edlén, $B$. Huang, $K$.

Edlén, $B$.

Huang, $K$.

Garstang, R. H.
Monthly Notices Roy. Astron. Soc. 84, 354-363

(1924).

Astrophys. J. 78, 239-297 (1927).

Naturwi ssenschaften $17,121-122$ (1928).

Astrophys. J. 69, $7-\overline{33}$ (1929).

Thesis Utrecht (1929) (same as below).

Arch. nèerl. sci. 3 A $12,1-75$ (1929) (same as above).

Pub1. Lick Observatory 17, 232-238 (1930).

Proc. Roy. Soc. London A 164, 167-191 (1938).

Phil. Trans. Roy. Soc. London. Ser. A. 242 $101-122$ (1949).

Astrophys. J. 111, 582-586 (1950).

Astrophys. J. $113,690=696$ (1951): [See al so Astron. J. 56, $38(1951-1952)$.]

Astrophys. J. 114, 469-472 (195i).

Proc. Roy. Soc. London A 208, $418-430$ (1951).

Astron. J. 56, 38 (1951-1952).

Z. Astrophys. 30, 275-281 (1952).

Doklady Akad. Nauk S.S.S.R. 98, 31-33 (1954) (trans1.).

Doklady Akad. Nauk Armyan. S.S.R. 20, 165-167 (1955) (transl.).

Optika i Spektroskopiya 1, 290-297 (1956) (transl.).

Soviet Phys. - Doklady 2, 167-169 (1957).

Optika i Spektroskopiya 4 , 421-429 (1958).

Bull. Acad. Sci. U.S.S.R. Phys. Ser. 22, 678-679 (1958).

Optika i Spektroskopiya 4, 430-437 (1958) (transl.).

Thes is Harvard (1958).

Bull. soc. roy. sci. Liège 30, 287-299 (1961).

Optics and Spectroscopy (U.S.S.R.) 10, 200-202 (1961).

Optics and Spectroscopy (U.S.S.R.) 10, 3-6 (1961) (see also below).

Optics and Spectroscopy (U.S.S.R.) 11, 307-309 (1961).

Submitted for publication, Proc. Cambridge Phil. Soc. (1962).

Ca VII

Astrophys. J. 114, 469-472 (1951).

Ca $X I 1$

Z. Astrophys. 22, 30-64 (1942).

Astrophys. J. 101, 187-195 (1945).

\section{$\mathrm{Ca} X\|\|$}

Z. Astrophys. 22, 30-64 (1942).

Astrophys. J. 101, 187-195 (1945).

Astrophys. J. 115, 569-570 (1952). misc。

estim.

quant.

misc. \& comm.

quant.

quant.

comm。\& estim.

SCF

SCF

SCF

SCF

quant. forb. comm.

SCF \& comm.

SCF

incompl.

incomp 1 .

hook, rel.

SCF \& estim. i ncomp I.

emiss., rel.

incompl. quant.

CA emiss., rel.

hook

comm.

SCF

quant. forb.

quant. forb. misc. forb.

quant. forb. misc. forb. quant. forb. 
Ca XV

Edlén, B.

Garstang, R. H. Garstang, R.'H.

Kuhn, W.

Kuhn, W.

Soleillet, P.

El lett, $A$.

Larche, K.

Zemansky, M. W.

Filippov, A. N.

Filippov, A. N.

Koenig, H. D. E Ellett, A.

Mitchel 1, A. C. G.

Soleillet, $P$.

Ornstein, L. S., van Hengstum,

J.P.A. E Brinkman, $H$.

King, G. W. E van Vleck, J. H.

King, R. B. E Stockbarger,

D. C.

Schuttevaer, J. W., de Bont, M.

J. E van den Broek, Th. H.

Webb, H. W. E Messenger, H. A.

Vainshtein, L. A. E Yavorskii,

B. M.

Matland, C. G.

Vainshtein, L. A. E Yavorskii, B. M.

van Hengstum, J.P.A.

van Hengstum, J.P.A. E

Smit, J.A.

Vainshtein, L. A.

Les, Z. E Ni ewodniczanski, H. Nagibina, I. M.

Addink, N. W. H.

Geneux, E. \& Wanders-Vincenz, B.

Penkin, N. P. E Red'ko, T. P.

Barrat, J.P. E Butaux, J.

Geneux, E. E Wanders-Vincenz, B.

Hey, P
Z. Astrophys. 22, 30-64 (1942).

Astrophys. J. 115, 569-570 (1952).

Proc. Cambridge Phil. Soc. 52, $107-113$ (1956).

$$
* * * * *
$$

Cd I

Naturwissenschaften $14,48-49$. (1926) (see also below).

Kgl. Danske Videnskab. Selskab Mat。-fys. Medd. Z, 1-86 (1926).

Compt. rend. 187, 212-214 (1928).

Phys. Rev. 33, 124 (1929).

Z. Physik 67, 440-477 (1931)。

Z. Physik $\frac{72}{72}, 587-599$ (1931).

Trudy Gosudarst. Opt. Inst. Leningrad 8 , \#77, 1-8 (1932) (transl.) (same as below).

Physik. Z. Sowjetunion 1, 289-296 (1932) (same as above).

Phys. Rev. 39, 576-584 (1932).

Phys. Rev. 43, 887-893 (1933).

Compt. rend.196, 1991-1993 (1933).

Physica 2, 145-152 (1938)。

Phys. Rev. 56, 464-465 (1939).

Astrophys. J. 21, 488-502 (1940).

Physica 10, 544-552 (1943).

Phys. Rev. 66, 77-86 (1944).

Doklady Akad. Nauk S.S.S.R. 87, 919-922 (1952) (transl.).

Phys. Rev. 91, 436 (1953).

Zhur. Eksptl. i Teoret. Fiz, 27, $712-718$ (1954) (SLA transi。 RT-3363).

Thesis Utrecht (1955) (see below for results).

Physica 22, 86-98 (1956).

Optika i Spektroskopiya 3, 313-321 (1957) (transl.).

Acta Phys. Polon. 17, 365-368 (1958).

Optika i Spektroskopiya 4, 430-437 (1958) (trans1.).

Spectrochim. Acta 15, 349-359 (1959).

Helv. Phys. Acta 33, 185-220 (1960) (transl.).

Optics and Spectroscopy (U.S.S.R.) 9, 360 (1960).

Compt. rend. 253, 2668-2670 (1961).

Cd 11

Helv. Phys. Acta 33, 185-220 (1960) (transl.). misc.

$* * * * *$

Cl

Z. Physik 157, 79-88 (1959). emiss.

quant. forb. quant. forb. quant. forb.

misc.

misc。

misc。

misc.

misc., rel. absorpt.

hook, rel.

hook, rel.

life

comm.

misc.

emiss., rel.

quant. forb., rel.

absorpt.

emiss., rel.

life

incompl.

life

quant.

emiss.

emiss.

incompl.

emiss., rel. incompl.

emiss.

misc.

hook, rel. E emiss., rel. misc. 
Osterbrock, D.E.

Hey, P.

Gaustad, J.E. E Spitzer, Jr., L.

Gaustad, J.E. \& Spitzer, Jr., L.

Bouma, $T$.

Ornstein, L. S. E Bouma, T. King, R. B., Parnes, B. R., Davis, M. H. E Olsen, K. H. Allen, C. W. E Asaad, A. S.

Hinnov, E. E Kohn, H. Ostrovskii, Yu. I. EPenkin, N. P.

Varsavsky, C.M. Addink, No W. H. Allen, C. W.

Frerichs, R.

Frerichs, R.

Allen, J. S. V. \& Hesthal, C. E.

Rozhestvenskii, D. S. E Penkin, N. P.

Rozhestvenski i, D. S. E Penkin, N. P.

Penkin, N.P.

Wilson, 0. C.

Estabrook, F.B.

Hill, A. J.

Estabrook, F.B.

Hill, A. J. E King, R. B. Huldt, L. E Lagerquist, A.

Estabrook, F.B. Huldt, L. E Lagerquist, A.

Davis, M. H., Routly, P. M. E. King, R. B.
Astrophys. J. 114, 469-472 (1951).

Z. Physik 157, 79=88 (1959).

Astrophys. J. 134, 771-776 (1961).

Cl \|\|

Astrophys. J. 134, $771-776$ (1961).

$* * * * *$

Co I

Thesis Utrecht (1930) (see below for results).

Phys. Rev. 36, 679-693 (1930).

J. Opt. Soc. Am. 45, 350-353 (1955).

Monthly Notices Roy. Astron. Soc. 117, 36-49 (1957).

J. Opt. Soc. Am. 47, 156-162 (1957).

optika i Spektroskopiya $5,345-353$ (1958)

(transl.).

Thesis Harvard (1958).

spectrochim. Acta 15, 349-359 (1959).

Monthly Notices Roy. Astron. Soc. 121, 299-332 (1960).

$* * * * *$

$\mathrm{Cr}$

2. Physik 31, 305-310 (1925)。

Ann. Physik 81, 807-845 (1926).

Phys. Rev. 47, 926-931 (1935).

Izvest. Akad. Nauk S.S.S.R. Ser. Fiz. 5, 97= 101 (1941) (transl.).

J.Phys. (U.S.S.R.) 2, $319=337$ (1941).

Zhur. Ekspt1. i Teoret. Fiz. 17, 355-365 (1947) (transl.).

Astrophys. J. 107, 126-150 (1948).

Thesis California Institute of Technology (1950). [See Astrophys. J. 113, 684-689 (1951) for results.]

Thesis California Institute of Technology (1950). [See Hill, A. J. E King, R. B., J. Opt. Soc. Am. 41, 315-321 (1951) for results.]

Astrophys. J. 113, 684-689 (1951). [See al so ibid. $115,571-572(1952)$.]

J. Opt. Soc. Am. 41, 315-321 (1951).

Arkiv Fysik 5, 91-95 (1951). [Results same as J. Opt. Soc. Am. 42, 142 (1952).]

Astrophys. J. 115, 571-572 (1952).

J. Opt. Soc. Am. 42, 142 (1952). [Results same as Arkiv Fysik 5, 91-95 (1951).]

Proceedings of the National Science Foundation Conference on Stellar Atmospheres, Indiana Univ. 47-49 (1954). quant. forb. emiss. estim.

estim.

emiss., rel. emiss., rel. absorpt., rel.

emiss.

emiss. hook, rel.

quant. emiss. comm. \& CA

emiss., rel. emiss., rel. emiss., rel.

hook, rel.

hook, rel. \& comm. hook, rel.

misc., rel. absorpt.

absorpt., rel.

absorpt.

absorpt., rel. emiss.

comm.

emiss.

absorpt. 
Mitrofanova, L. A.

Bell, G. D.

Nikonova, E. 1. E Prokof'ev, V. K.

Allen, C. W. E Asaad, A. S. Hinnov, E. E Kohn, H.

Ostrovskii, Yu. 1. EPenkin, N.P.

Dvornikova, I. U. E Nagibina, I. M.

Addink, N. W. H.

Allen, C. W.

Boyarchuk, M. E. E Boyarchuk, A. A.

Wilkerson, T. D.

Allen, J. S. V. E Hesthal, C. $E$.

Penkin, N. P.

Wilson, 0. C.

Zagorianskaya, E. U.

Aller, L. H., Hazen, M., Doherty, L., Grant, G., Jugaku, Jo, Spiegel, E. \& Waddell, J.

Boyarchuk, M. E. \& Boyarchuk, A. A.

Groth, H. G.

Wilkerson, T. D.
Pulkovo. Astronomicheskaia observatoriia. Izvestila 19, \#152, 100-102 (1954) (trans1.).

Thesis Callifornia Institute of Technology (19561957).

Optika i Spektroskopiya 1, 290-297 (1956) (transl.).

Monthly Notices Roy. Astron. Soc. 117, 36-49 (1957). J. Opt. Soc. Am. 47, 156-162 (1957).

optika i Spektroskoplya 3, 193-201 (1957) (transl.).

Optika i Spektroskopiya 4, 421=429 (1958).

spectrochim. Acta 15, 349-359 (1959).

Monthly Notices Roy. Astron. Soc, 121, 299-332 (1960).

Akademi ia Nauk S.S.S.R. Krymskaia astrofizecheskaia observatorila. Izvestila 22, 234-256 (1960) (transl.).

Thesis Michigan (1961).

\section{$\mathrm{Cr} \|$}

Phys. Rev. 47, 926-931 (1935).

Zhur. Eksptl。i Teoret. Fiz, 17, 1114-1121 (1947) (transl.).

Astrophys. J. 107, 126-150 (1948).

zhur. Ekspt1. i Teoret. Fiz. 19, 447-450 (1949) (transi.).

Publications of the Observatory, Univ. of Michigan (Feb. 19, 1954).

Akademila Nauk S.S.S.R. Krymskaia astrofizecheskaia observatorila. Izvestila 22, 234-256 (1960) (transl.).

Z. Astrophys. 51, 231-285 (1961).

Thesis Michigan (1961).

$$
* * * * *
$$

\section{Cs 1}

Ann. Physik 43, 96-134 (1914).

Physik. Z. 21, 322-324 (1920).

Physik. Z. $23,73=80$ (1922).

Phys. Rev。 23, 693-709 (1924).

Thesis Utrecht (1928).

Z. Physik 27, 387-393 (1929). [Same as Zhur. Ekspt1. i Teoret. Fiz. 1, 123-127 (1931).]

Z. Physik 53, 459-482 (1929).

z. Physik 63, 198-209 (1930).

Proc. Phys.-Math. Soc. Japan 12, 93=100 (1930).

Z. Physik 64, 682-696 (1930).

Zhur. Eksptl。i Teoret. Fiz. 1, 123-127 (1931) (transl.). [Same as Z. Physik 57, 387-393 (1929).]

Zhur. Eksptl。i Teoret. Fiz, 2, 24-41 (1932) (transl.).

Physica $1,945=952$ (1934).

Zhur. Eksptl. i Teoret. Fiz. 4, 359-367 (1934) (transl.).

Thesis Utrecht (1937).

Thesis Utrecht (1937). emiss., rel.

absorpt.

hook, rel.

emiss.

emiss.

hook

incompl.

emiss.

comm. \& CA

comm.

emiss.

emiss, rel.

hook, rel.

misc., rel. incompl.

misc, rel.

comm .

misc.

emiss. absorpt, rel. absorpt.

absorpt.

comm.

emiss., rel. hook forb., rel.

absorpt.

misc.

quant.

absorpt., rel. hook forb., rel.

hook, rel.

comm., rel. hook, rel.

emiss.

emiss., rel. 
Kvater, G. S. \& Meister, T. G.

Bersuker, I.B.

Vainshtein, L.A.

Archambault, $Y_{0}$, Descoubes, $J_{0}$,

Priou, M., Omont, A. E Pebay-

Peyroula, J.

Hawkins, W. B.

Heavens, 0 . S.

Naqvi, A. M.
Al thoff, $K$.

Vestnik Leningrad Univ. Ser. Fiz, i Khim. I, $137-158$ (1952) (transl.).

z. Physik 141, 33-42 (1955).

Soviet Phys. = Doklady 2, 167-169 (1957).

Optika i Spektroskopiya $3,313-321$ (1957) (transi.).

J. phys. radium 21, 677-679 (1959).

Phys. Rev. 123, 544-547 (1961).

J. Opt. Soc.Am. 51, 1058-1061 (1961).

Geophysics Corporation of America Technical

Report 61-21-A; ASTIA Document 263459 (1961).

$$
* * * * *
$$

Cu

van Lingen, $D$.

van Lingen, $D$.

King, R. B. E Stockbarger,

D. C.

Schuttevaer, J.W., de Bont,

M. J. E van den Broek, Th. H.

van den Bold, H. J.

Davis, M. H., Routly, P. M.

E King, R. B.

Parchevskii, G. F. E Penkin, N.P.

Bell, G. D.

Allen, C. W. E Asaad, A. S. Bell, G. D.

Ostrovskii, Yu. I. E Penkin, N. P.

Bell, G. D., Davis, M. H.,

King, R. B. E Routly, P.M. Varsavsky, C. M. Addink, N. W. H.

Vidale, G. L.

Garstang, R. H.

Garstang, R. H.

Yilmaz, $H_{0}$

Bolotin, A. B., Levinson,

I. B. E Levin, L.I.

Bolotin, A. B. E Yutsis, A. P.

Cs I to F V
Thes is Utrecht (1936) (see below for results).

Physica 3, 977-994 (1936)。

Astrophys. J. 91, 488-502 (1940). [See also

Bell, G. D., Astrophys. J. 127, 775-796 (1958).]

Physica 10, 544-552 (1943).

Thes is Utrecht (1945).

Proceedings of the National Science Foundation

Conference on Stellar Atmospheres, Indiana

Univ., 47=49 (1954).

Soviet Phys. - JETP $1,382-384$ (1955).

Thesis California Institute of Technology (1956-

1957). [See Bell, G. D., et al., Astrophys. J.

127, 775-796 (1958) for results.]

Monthly Notices Roy. Astron. Soc. 117, 36-49 (1957).

Astron. J.62, 7 (1957). [See Bell, G. D., et al.,

Astrophys.J. 127, 775-796 (1958) for results.]

optika i Spektroskopiya $3,193-201$ (1957) (transl.).

Astrophys. J. 127, 775-796 (1958).

Thesis Harvard (1958).

Spectrochim. Acta 15, 349-359 (1959).

Technical Information Series Report R60SD331, General Electric Co., Space Science Laboratory (1960).

$$
* * * * *
$$

$F \|$

Monthly Notices Roy. Astron. Soc. 111, 115-124 (1951).

\section{$F \mid V$}

Monthly Notices Roy. Astron. Soc. $111,115-124$ (1951).

Phys, Rev, 100, 1148-1153 (1955).

Soviet Phys。 JETP 2, 391-395 (1956).

\section{F V}

Zhur. Eksptl.i Teoret. Fiz. 24, 537-543 (1953) (transl.).

$$
* * * * *
$$

14 hook

misc. estim. incompl. misc.

quant.

CA

CA

emiss., rel. emiss., rel. absorpt.

emiss., rel.

emiss., rel. absorpt.

hook, rel.

absorpt.

emiss.

comm.

hook

absorpt.

quant. emiss. absorpt.

quant. forb.

quant. forb. quant. forb. quant. 
van Milaan, J. B.

Frerichs, R.

van Milaan, J. B. van Milaan, J. B.

Russel1, H. N.

Allen, C. W.

King, R. B. $\&$ King, A. S。

van Driel, $H$.

Menzel, D. H. E Goldberg, L.

King, R. B. E King, A. S.

Rozhestvenskii, D. S. E Penkin,

N. P.

Rozhestvenskii, D. S. \& Penkin, N. P.

King, R. B.

Sobolev, N. N.

Petrie, W.

Gottschalk, W. M.

Carter, W. W.

Carter, W. W.

Crosswhite, H. M.

Kopfermann, H. \& Wessel, G.

Mi trofanova, L. A.

Aarts, J., Harting, D. E Bakker, C. J.

Parchevski, G. F. E Penkin, N. P.

Bell, G. D.

Osberghaus, O. E Ziock, K.

Allen, C. W. E Asaad, A. S.

Bell, G. D.

Hinnov, E. E Kohn, H.

Ziock, K.

Bell, G. D., Davis, M. H., King, R. B. E Routly, P. M. Crosswhite, H. M.

Mel'nikov, 0. A.

Nagibina, 1. M.

Nagibina, 1. M.

Addink, N. W. H.

Hefferlin, R.

Hefferlin, R.

Zwaan, A.

Allen, C. W.
Z. Physik 34, 921-923 (1925).

Ann. Physik 81, 807-845 (1926).

Thesis Utrecht (1926) (see below for results).

Z. Physik 38, 427-439 (1926).

Astrophys. J. 78, 239-297 (1927).

Monthly Notices Roy. Astron. Soc. 96, 145-148 (1935).

Astrophys. J. 82, 377-395 (1935). [See al so Menzel, D. H. E Goldberg, L., Astrophys. J. 85, 40-41 (1936) and King, R. B. E King, A. S., Astrophys. J. 87, 24-39 (1938).]

Thesis Utrecht (1935).

Astrophys. J. $85,40-41$ (1936).

Astrophys. J. 87, 24-39 (1938).

Izvest. Akad. Nauk S.S.S.R. Ser. Fiz. 5, 97-101

(1941) (transl.) (see al so below).

J. Phys. (U.S.S.R.) 5, 319-337 (1941).

Astrophys. J. 95, 78-81 (1942).

Zhur. Eksptl. i Teoret. Fiz. 13, 131-136 (1943) (transl.).

Can. J. Research A 25, 42-48 (1947).

As trophys. J. 108, 326-337 (1948).

Thes is California Institute of Technology (1949) (see below for results).

Phys. Rev. 76, 962-966 (1949).

Spectrochim. Acta 4, 122-151 (1950).

Z. Physik 130, 100-108 (1951).

Pulkovo. Astronomicheskaia observatori ia. Izvestila 19, \#149, 81-139 (1952) (trans1.).

Physica 20, 1250-1258 (1954)。

Bull. Acad. Sci. U.S.S.R. Phys. Ser. 19, 4-5 (1955).

Thesis California Institute of Technology (19561957). [See Bell, G. D., et al., Astrophys.J. $127,775-796$ (1958) for results.]

z. Naturforsch. 1la, 762-763 (1956). [See al so Ziock, K., Z. Physik 147, 99-112 (1957).]

Monthly Notices Roy. Astron. Soc. 117, 36-49 (1957).

Astron. J. 62, 7 (1957). [See Bell, G. D., et al., Astrophys.J. 127, 775-796 (1958) for results.]

J. Opt. Soc. Am. 47, 156-162 (1957).

2. Physik 147, 99-112 (1957). [See also Ottinger, C. \& Ziock, K., Z. Naturforsch. 16a, 720 (1961).]

Astrophys. J. 127, 775-796 (1958).

Johns Hopkins Spectroscopic Report No. 13 (1958).

Pulkovo. Astronomicheskaia observatorila. Izvesti ia 20, \#6, 28-43 (1958) (transl.).

Bull. Acad. Sci. U.S.S.R. Phys. Ser. 22, 678-679 (1958)

Optika i Spektroskopiya 4, 430-437 (1958) (transl.). Spectrochim. Acta 15, 349-359 (1959).

J. Opt. Soc. Am. $49,680-685$ (1959) (see al so below).

J. Opt. Soc. Am. 49, 948-949 (1959). [See al so Leftus, $V_{0}$, Central Astronomical Institutes of Czechoslovakia: Bulletin 12, 161-167 (1961).]

Bulletin Astronomical Institutes Netherlands 14 , 288-298 (1959).

Monthly Notices Roy. Astron. Soc. 121, 299-332 (1960). emiss., rel. emiss., rel. emiss., rel. emiss., rel. estim.

emiss.

absorpt., rel.

emiss., rel.

comm.

absorpt., rel.

hook, rel.

hook, rel. \& comm.

absorpt.

emiss., rel.

comm.

quant., rel. emiss., rel.

emiss, rel. emiss., rel. absorpt.

emiss., rel.

emiss., rel.

comm.

absorpt.

life

emiss.

comm.

emiss.

life

absorpt.

compil.

incompl.

emiss., rel.

i ncomp 1 .

emiss.

emiss., rel.

emiss., rel.

misc., rel.

comm. \& CA 
Boyarchuk, M. E. E Boyarchuk, A. A.

Belousova, I. M. E Gurevich, D. B.

Korolev, F. A. \& Kvaratskheli, Yu. K.

Leftus, $V$.

Leftus, $V$.

Ottinger, C. E Ziock, K.

Jefferies, J. T. E Orrall, F. Q.
Akademila Nauk S.S.S.R. Krymskaia astrofizecheskaia observatorila. Izvestila 22, 234-256 (1960)

(transl.).

Optics and Spectroscopy (U.S.S.R.) 10, 206-207 (1961).

Optics and Spectroscopy (U.S.S.R.) 10, 200-202 (1961).

Central Astronomical Institutes of Czechoslovakia: Bulletin 12, 161-167 (1961).

J. Opt. Soc. Am. 51, 1151 (1961) (see also above).

Z. Naturforsch. 16a, 720 (1961).

Astrophys. J. 135, 109-121 (1962).

\section{$\mathrm{Fe} \|$}

Russell, H. N. Clarkson, W. Rubinowicz, A.

Sobolev, N. N.

Crosswhite, H. M.

Mi trofanova, L. A.

Aller, L. H., Hazen, M. Doherty, L., Grant, G., Jugaku, J., Spiegel, E. E Waddell, J.

Mel'nikov, 0. A.

Boyarchuk, M. E. E Boyarchuk, A. A.

Groth, H. G.

Garstang, R. H.

Gaustad, J.E. E Spitzer, Jr., L.

Garstang, R. H.

Garstang, R. H.

Edlén, B.

Huang, K.

Pecker, C.

Garstang, R. H.
Astrophys. J. 78, 239-297 (1927).

Phi 1. Mag. I, 98-105 (1929).

Z. Physik 65, 662-676 (1930).

Zhur. Eksptl. i Teoret. Fiz. 13, 131-136 (1943)

(transl.).

spectrochim. Acta 4, 122-151 (1950).

Pulkovo. Astronomicheskaia observatorila. Izvestila 19. \#151, 45-48 (1953).

Publications of the Observatory, Univ. of Michigan (Feb. 19, 1954).

Soviet Astronomy - AJ 3, 381-388 (1959).

Akademi ia Nauk S.S.S.R. Krymskaia astrofizecheskaia observatorila. Izvestila 22, 234-256 (1960) (transl.).

Z. Astrophys. 51, 231-285 (1961).

\section{Fe \|\|}

Monthly Notices Roy. Astron. Soc. 117, 393-405 (1957).

Astrophys. J. 134, $771=776$ (1961).

$\mathrm{Fe} \| \mathrm{V}$

Monthly Notices Roy. Astron. Soc. 118, 572-584

(1958).

Fe V

Monthly Notices Roy. Astron. Soc. 117, 393-405

(1957).

Fe $X$

Z. Astrophys. 22, 30-64 (1942).

Astrophys. J. 101, 187-195 (1945).

Compt. rend. 250, 3779-3781 (1960).

Submitted for publication, Ann. astrophys. (1962). comm.

comm.

emiss., rel.

emiss. \& comm.

comm.

1 ife

estim.

estim.

emiss., rel. quant., rel. emiss., rel.

emiss., rel. emiss., rel.

misc, rel.

misc. $E$ misc。 forb., rel. comm.

misc。

quant. forb.

estim.

quant. forb.

quant. forb.

quant. forb. misc. forb. misc. comm. 
Fe $X I$

Edlén, B. Huang, $K$.

Edlén, B. Huang, $K$.

Edlén, B. Gold, M. T.

Froese, C.

Garstang, R. H.

Edlén, B.

Osterbrock, D. E.

Blaha, M. Huang, $K$.

Z. Astrophys. 22, 30-64 (1942).

Astrophys. J. 101, 187-195 (1945).

Fe $X\|\|$

Z. Astrophys. 22, 30-64 (1942).

Astrophys. J. 101, 187-195 (1945).

Fe XIV

Z. Astrophys. 22, 30-64 (1942).

Astrophys. J. 101, 187-195 (1945).

Monthly Notices Roy. Astron. Soc. 109, 471-

477 (1949).

Monthly Notices Roy. Astron. Soc. 117, 615-621

(1957).

Submitted for publication, Ann. astrophys. (1962).

Fe XV

Z. Astrophys. 22, 30-64 (1942).

Astrophys. J. $114,469-472$ (1951).

Central Astronomical Institutes of Czechoslovakia: Bulletin 8, 34-37 (1957).

$* * * * *$

Ga
Allen, C. W.

Allen, C. W. E Asaad, A. S.

Ostrovskii, Yu. I. E Penkin, N. P.

Ostrovskii, Yu. 1., Penkin,

N. P. E Shabanova, L. N.

Brehm, B., Demtröder, W. E Osberghaus, 0 .

Demtröder, $W$.

Hanus, W.

Ottinger, C. E Ziock, K. Demtröder, $W$.

Monthly Notices Roy. Astron. Soc. 117, 622-628

(1957).

Monthly Notices Roy. Astron. Soc. 117, 36-49

(1957) (see al so above).

Optika i Spektroskopiya 4, 719-724 (1958) (transl.) hook (results same as below).

Bull. Acad. Sci. U.S.S.R. Phys. Ser. 22, 720-724 hook (1958) (results same as above).

Z. Naturforsch. 16a, 843 (1961). [See also Demtröder, $W_{.}$, below and Z. Physik 166, 42-45 (1962).]

Thesis Bonn (1961). [See Z. Physik 166, 42-45 (1962) for results.]

Bull. acad. polon. sci. Classe 3, 8, 629-636 (1961).

Z. Naturforsch, 16a, 720 (1961).

Z. Physik 166, 42-45 (1962).

$* * * * *$

H I

*Hoyt, F. C.

*Slack, F。G.

*Crew, W. H. E Hulburt, E. 0 . *Russell, H. N.

Sugiura, M. Y.

*Wien, W. quant. forb. misc. forb.

quant. forb. misc. forb.

quant. forb. misc. forb. SCF

SCF

quant. forb.

quant. forb. quant. forb. quant. forb.

comm.

emiss.

life

life

comm.

life

life

quant., rel.

life

emiss., rel. estim.

quant.

canal 
*arst, A. E Ladenburg, R.

*Kupper, A.

*Slack, F。G.

*Snoek, J. L. E Ornstein, L. S.

Sugiura, $Y$.

*Menzel, D. H.

Stevenson, A. F.

*Maxwel 1, L. R.

*Griffiths, J. H. E。

*Woolley, R.

Menzel, D. H. E Pekeris, C. L.

*Rudnick, P.

Breit, G. E Teller, E.

Biermann, $L$.

*Veselov, M. G.

Wild, J.P.

Harriman, J. M.

Bethe, H. A. E Salpeter, E. E.

Green, L. C., Rush, P.P.E Chandler, C. D.

Fite, W. L., Brackmann, R. T., Hummer, D. G. E Stebbings, R. F.

Shapiro, J. E Breit, G.

Demidov, V. P.

Herdan, R. $\varepsilon$ Hughes, T. P.

Herdan, R. \& Hughes, T.P.

Karzas, W. J. \& Latter, R.

\author{
*Russel1, H. N. \\ *Levy, S. \\ Vinti, J.P. \\ Wheeler, J. A. \\ Korwein, $H$. \\ Hylleraas, E. A. \\ Gol dberg, L. \\ Breit, G. \& Teller, E. \\ Goldberg, L. \\ Romberg, $W$. \\ Huang, Su-Shu \\ Bates, D. R. \& Damgaard, A. \\ Veselov, M. G. \\ Wellmann, P. \\ Veselov, M. G. \\ Heron, So, McWhirter, R. W. P. \\ \& Rhoderick, E. H. \\ Heron, S., McWhirter, R. W. P. \\ \& Rhoderick, E. H. \\ Miller, W. F. \\ Vizbaraite, Ya. I., \\ Kantserevichyus, A. I. \& \\ Yutsis, A. P. \\ Yutsis, A. P., Ushpalis, K. K., \\ Karetskis, V. I. E Levinson, \\ I. B.
}

Z. Physik 48, 192-204 (1928)。

Ann. Physik 86, $511-529$ (1928).

Phys. Rev. $31,527=532$ (1928).

Z. Physik 50, 600-608 (1928).

Science Papers of the Institute of Physical and Chemical Research 11, 1-80 (1929).

Publ. Lick Observatory 17, 232-238 (1930).

Proc. Roy. Soc. London A 128, 591-599 (1930).

Phys. Rev. 38, 1664-1686 (1931).

Proc. Roy. Soc. London A 147, 547-554 (1934).

Monthly Notices Roy. Astron. Soc. 95, 101-116 (1934).

Monthly Notices Roy. Astron. Soc. 26, 77-111 (1935).

Phys. Rev. 48, 807-811 (1935).

Astrophys. J. 91, 215-238 (1940).

Physik. Z. 44, 232-233 (1943)

Zhur. Ekspt1. i Teoret. Fiz. 19, 959-964 (1949) (ATS transl.).

Astrophys. J. 115, 206-221 (1952).

Phys. Rev. 101, 594-598 (1956).

"Quantum Mechanics of One and Two Electron Atoms"

(Academic Press, New York, 1957).

Astrophys. J. Suppl. Ser. 3, 37-50 (1957).

Phys. Rev. 116, 363-367 (1959).

Phys. Rev. 113, 179-181 (1959).

Astronomicheskii Zhurnal 38, 1065-1068 (1961).

Astrophys. J. 133, 294-298 (1961) (see al so below).

Astrophys. J. 134, 1028 (1961).

Astrophys. J. Suppl. Ser. 6, 167-212 (1961).

$$
* * * * *
$$

He I

Astrophys. J. 78, 239-297 (1927).

Z. Physik $72,578-586$ (1931).

Phys. Rev. 42, 632-640 (1932).

Phys. Rev. 43, 258-263 (1933).

2. Physik 91, $1-36$ (1934)

2. Physik 106, 395-404 (1937).

Astrophys. J. 90, 414-428 (1939).

Astrophys. J. 91, 215-238 (1940).

Astrophys. J. 23, 244-249 (1941).

Phys. Rev. 71, 706-711 (1947).

Astrophys. Jo 108, 354-372 (1948).

Phil. Trans. Roy. Soc. London. Ser. A. 242 $101-122$ (1949).

Zhur. Eksptl \& Teoret. Fiz. 19, 959-964 (1949) (ATS transl.).

Z. Astrophys. 30, 71-87 (1952).

Vestnik Leningrad Univ. Ser. Fiz. Khim. No. 8, 181-185 (1953) (trans1.).

Nature 174, 564-565 (1954) (see also below).

Proc. Roy. Soc. London A 234, 565-582 (1956).

Thesis Purdue (1956).

Optika i Spektroskopiya 1, 9-16 (1956) (transl.).

Optika i Spektroskopiya 1, 601-605 (1956)

(transl.). hook, rel.

quant.

quant.

absorpt., rel.

quant.

comm. E estim. quant. forb.

comm.

life

comp i 1 .

quant.

quant.

quant. forb.

quant.

quant.

quant.

quant.

compil.

quant.

life forb.

quant. forb. quant. forb. quant.

comm.

quant.

estim.

hook, rel.

quant.

quant.

quant.

quant.

quant.

quant. forb.

quant. forb.

SCF

comm.

$\mathrm{CA}$

quant.

estim.

quant.

life

life

comm.

SCF

quant. 
Dalgarno, A. E Lynn, N. Miller, W. F. E Platzman, R. L.

Trefftz, E., Schluter, A., Dettmar, K. H. \& Jörgens, K.

Dalgarno, A. E Kingston, A. E.

Mastrup, F. E Wiese, W. Osherovich, A. L. E Savich, I. G.

*Varsavsky, C. M. Aller, L. H. E Jugaku, J. Bennett, R. G. E Dalby, F. W.

Dalgarno, A. \& Stewart, A. L.

Decomps, B., Pebay-Peyroula, J. C. E Brossel, J.

McLean, E. A.

Salpeter, E. E. E Zaidi, M. $H$.

Maxwel I, L. R.

Füch tbauer, C. \& Joos, G. Fưchtbauer, C., Joos, G. \& Dinkelacker, 0 .

Tolman, R. C.

Wien, $W$.

Skinner, H. W. B.

Trumpy, B.

Hughes, A. L. E Thomas,

A. R.

Trumpy, B.

Kunze, $P$.

Gaviola, E.

Kopfermann, H. E Tietze, W. Ladenburg, $R$.

Webb, H. W. \& Messenger, H. A.

Ladenburg, R. \& Wolfsohn, G. Ladenburg, R. \& Wol fsohn, G. Randall, R. H.

Richter, E. F.

Thomas, A. R.

Garrett, P. H. \& Webb, H.W. Garrett, P. H.

Mitchell, A. C. G.
Proc. Phys. Soc. London A 70, 802-808 (1957).

Proc. Phys. Soc. London A $70,299-303$ (1957).

2. Astrophys. 44, 1-17 (1957).

estim.

comm.

SCF

Proc. Phys. Soc. London A 72, $1053-1060$ (1958).

estim.

Z. Astrophys. 44, 259-279 (1958).

Optika i Spektroskopiya 4, 715-718 (1958)

(transl.).

Thesis Harvard (1958).

Astrophys. J. Suppl. Ser. 4, \#38, 109-156 (1959).

J. Chem. Phys. 31, 434-441 (1959).

Proc. Phys. Soc. London A 76, 49-55 (1960).

Compt. rend. 251, 941-943 (1960) (transl.).

"Fourth Symposium on Temperature: Its Measurement and Control in Science and Industry"

(ed. Herzfeld, C. M., Reinhold Publ., New York, to be published).

Phys. Rev. 125, 248-255 (1962).

emiss., rel. incompl.

quant.

comm.

life

estim.

misc.

emiss.

quant.

$\mathrm{He} \|$

Phys. Rev. 38, 1664-1686 (1931).

life

$$
* * * \dot{*} *
$$

$\mathrm{Hg} \quad$

Physik. Z. 23, 73-80 (1922).

Ann. Physik Z1, 204-227 (1923).

Phys. Rev. 23, 693-709 (1924).

Ann. Physik 73, 483-504 (1924).

Proc. Roy. Soc. London A 112, 642-660 (1926).

Z. Physik 40, 594-617 (1926).

Phys. Rev. $30,466-472$ (1927).

Kgl. Norske Videnskab. Selskabs, Skrifter 1 , 1-80 (1927).

Ann. Physik 85, 1013-1057 (1928).

Contribucion al Estudio de las Ciencias. Fisicas y Matematics, La Plata, Argentina 5 , 65-75 (1929). Universidad Nacional de la Plata Publicaciones de la Facultad de Ciencias Fisicomatematics (transl.).

Z. Physik 56, 604-616 (1929).

Nachr. Akad. Wiss. Göttingen, Math.-physik. Kl. $1=5$ (1929).

Phys. Rev. $33,319-328$ (1929).

2. Physik 63, 616-633 (1930).

Z. Physik $\frac{65}{65}, 207-208$ (1930).

Phys. Rev. 35, 1161-1169 (1930).

Ann. Physik 7, 293-328 (1930).

Phys. Rev. 35, 1253-1261 (1930).

Phys. Rev. 37,1686 (1931).

Phys. Rev. $\frac{40}{43}, 779-790$ (1932).

Phys. Rev. $43,887-893$ (1933). hook

life

absorpt. absorpt.

comm. canal

misc.

comm.

absorpt.

absorpt.

absorpt. comm. forb.

hook

comm.

life

misc. absorpt.

life

life

comm. 
Wolfsohn, $G$.

Wol fsohn, G.

Mitchel1, A. C. G. E Murphy, E.J.

Mitchel 1, A. C. G. E Murphy, E. J.

Opechowski, W.

King, G. W. E van Vleck, J.H.

Schouten, J.W.

Schouten, J. W.

Schouten, J. W. E Smit, J.A.

Lennuier, R. \& Cojan, J.L.

Mishra, B.

Brossel, J.

Brossel, J.

Brossel, J.

Brossel, J. E Bitter, F.

Mishra, B.

Vainshtein, L. A. E Yavorskii, B. M.

Vainshtein, L. A. E Yavorski $i$, B. M.

Brannen, E., Hunt, F. R., Adlington, R. H. E Nicholls, R. W.

Lebedeva, V. V. E Fabrikant, V. A.

Guiochon, M. A., Blamont, J. E. \& Brossel, J.

Barrat, J.P.

Boutron, F., Barrat, J.P. $E$ Brossel, J.

Guiochon, M. A., Blamont, J. E. E Brossel, J.

Vainshtein, L. A.

Descoubes, J.P. \& Pebay-

Peyroula, J.C.

Strijland, J. C. E Nanassy, A. J.

Addink, N. W. H.

Barrat, J. P.

Cojan, J. L. E Thibeau, M.

Pebay-Peyroula, J. C.

Koedam, M. E Kruithof, A. A.

Maxwel 1, L. R.

Maxwell, L. R.
Z. Physik 83, 234-247 (1933).

Z. Physik 85, 366-372 (1933).

Phys. Rev. 45, 759 (1934) (see also below).

Phys. Rev. 46, 53-58 (1934).

Z. Physik 109, 485-490 (1938).

Phys. Rev. 56, 464-465 (1939).

Thesis Utrecht (1941). [See Schouten, J. W. E Smit, J.A., Physica 10, 661-671 (1943) for results.]

Physica 10, 672-678 (1943).

Physica 10, 661-671 (1943).

Compt. rend. 231, 1450-1452 (1950).

Phys. Rev.77, 153 (1950).

Phys. Rev. 83, 210 (1951) (see al so below).

Thesis Paris (1952) (see also below).

Ann. phys. Z, 622-627 (1952) (see also below).

Phys. Rev. 86, 308-316 (1952). [See also Brossel, J., Phys. Rev. 83, 210 (1951).]

Proc. Cambridge Phil. Soc. $48,511-515$ (1952).

Dokl ady Akad. Nauk S.S.S.R. 87, 919-922 (1952) (transl.).

Zhur. Eksptl. i Teoret. Fiz. 27, 712-718 (1954) (SLA transl. RT-3363).

Nature 175, 810-811 (1955).

Bull. Acad. Sci. U.S.S.R. Phys. Ser. 19, 2-4 (1955).

Compt. rend. 243, 1859-1862 (1956).

Compt. rend. 244, 2785-2788 (1957) (trans1.).

Compt. rend. 245, 2250-2253 (1957).

J. phys. radium 18, 99-108 (1957).

Optika i Spektroskopiya 3, 313-321 (1957) (transl.). Compt. rend. 247, 2330-2332 (1958) (transl.).

Physica 24, 935-936 (1958).

Spectrochim. Acta 15, 349-359 (1959).

J. phys. radium 20, 657-668 (1959).

Compt. rend. 249, 1489-1491 (1959) (transl.).

J. phys. radium 20,721-729 (1959).

Physica 28, 80-100 (1962).

$\mathrm{Hg} \mathrm{II \|}$

Phys. Rev. 34, 199-206 (1929).

life

$\mathrm{Hg}$ IV

Phys. Rev. 34, 199-206 (1929).

life hook

hook

misc.

misc.

comm. forb. quant. forb., rel.

emiss.

emiss., rel. emiss.

misc.

SCF

misc.

misc.

misc.

misc.

SCF

i ncomp 1 .

SCF

life

emiss., rel.

E quant. rel.

misc.

misc.

misc.

misc.

incompl. misc.

comm.

emiss. misc.

misc.

misc.

emiss. 

(1960).

$$
* * * * *
$$

$\ln \mid$

Ostrovskii, Yu. I. E Penkin, N. P.

Ostrovskii, Yu. I., Penkin, N. P. E Shabanova, L. N. Ostrovskii, Yu. I., Penkin, N. P. E Shabanova, L. N. Vainshtein, L. A.

Ch'en, S. Y. \& Smith, A. Hanus, W.

Rupp, E.

Ditchburn, R. W.

Prokof'ev, V. K. E Gamow, G.

Bl eeker, C. E.

Lawrence, E. O. E Edlefsen, N. E.

Prokof'ev, V. K.

Weiler, J.

Stevenson, A. F.

Prokof'ev, V. K.

Filippov, A. N.

Filippov, A. N.

Filippov, A. N.

Ornstein, L. S. E Key, J.

Prokof'ev, V. K.

van der Held, E. F. M. E

Heierman, J. H.

Coenen, P. A. E Kramers, H. A.

Sinclair, D. E Webb, H.W.

van der Held, E. F. M. E

Hei erman, J.H.

Heierman, J. H.

Key, J.

Schwarz, K. H.

Rozhestvenskii, D. S. E Penkin, N. P.
Optika i Spektroskopiya 4, 719-724 (1958)

(transl.) (see below for results).

Bull. Acad. Sci. U.S.S.R. Phys. Ser. 22 , $720-724$ (1958).

Soviet Phys. - Doklady 3, 538-540 (1958).

Bull. Acad. Sci. U.S.S.R. Phys. Ser. 22 668 $=669$ (1958).

Physica 25, 1289-1293 (1959).

Bull. acad. polon. sci. Classe 3, $8,629-636$ (1961).

\section{$* * * * * *$}

\section{K I}

Ann. Physik 80, 524-532 (1926).

Proc. Roy. Soc. London A $117,486-508$ (1927).

Z. Physik 44, 887-892 (1927).

Thesis Utrecht (1928).

Phys. Rev. 34, 1056-1060 (1929)。

Z. Physik 57, 387-393 (1929). [Same as Zhur. Eksptl. i Teoret. Fiz. 1, 123-127 (1931).]

Ann. Physik 1, 361-399 (1929).

Proc. Roy. Soc. London A 128, 591-599 (1930).

Zhur. Eksptl. i Teoret. Fiz. 1, 123-127 (1931)

(transl.). [Same as Z. Physik 57, 387-393 (1929).]

Zhur. Eksptl. i Teoret. Fiz. 2, 24-41 (1932) (transl.).

Physik. Z. Sowjetunion 5, 1-5 (1933) (same as below).

Zhur. Eksptl. i Teoret. Fiz. 3, 520-523 (1933) (transl.) (same as above).

Physica 1, 945-952 (1934).

Zhur. Eksptl. i Teoret. Fiz. 4, 347-358 (1934) (transl.).

Physica 2, 71-74 (1935). [See also ibid. 3, $31-41 \quad(1936)$.

Physica 3, 341-345 (1936).

Phys. Rev. 50, 440-445 (1936).

Physica 3, 31-41 (1936).

Thesis Utrecht (1937).

Thesis Utrecht (1937).

Physica Z, 361-368 (1940).

I zvest. Akad. Nauk S.S.S.R. Ser. Fiz. 5, 97-

101 (1941) (transl.). hook

hook

hook

SCF

absorpt. quant.

canal

absorpt. hook, rel. emiss., rel. \& hook, rel. absorpt.

hook forb., rel.

misc., rel. quant. forb. hook forb., rel.

incompl.

hocik, rel. hook, rel.

emiss., rel. hook, rel.

emiss., rel.

quant.

life

emiss.

emiss. emiss., rel. emiss. hook, rel. 
Rozhestvenskii, D. S. E Penkin, N. P.

Bi ermann, L.

Biermann, L.

Bi ermann, L.

Bi ermann, L. E Lübeck, K.

Sobolev, N. N.

Mishra, B.

Stephenson, G.

Stephenson, G.

villars, D. S.

Vainshtein, L. A. \& Yavorski i,

B. M.

Yanoukh, F。

Bersuker, I. B.

Hinnov, E. E Kohn, H.

Ritter, G. J. E Series, G. W. Varsavsky, C.M.

Addink, N. W. H.

Heavens, 0. S.

Houziaux, L. E Sadoine, M. P.

Naqvi, A. M.

Osterbrock, D. E.

Osterbrock, D. E.

Petersen, R.

Garton, W. R. S., Pery, A. \& Codling, $K$.

Pery-Thorne, A. E Garton, W. R. S .

Osterbrock, D. E.
J. Phys. (U.S.S.R.) 5, 319-337 (1941).

Physik. Z. 44, 232-233 (1943) (see also below).

Z. Astrophys. 22, 157-164 (1943) (see also below).

Nachr. Akad. Wiss. Göttingen, Math. -physik. KI.

$116=118(1946-1948)$ (see also below).

2. Astrophys. 25, 325-339 (1948).

Zhur. Eksptl. i Teoret. Fiz. 19, 25-35 (1949)

(transl.).

Phys. Rev. 27, 153 (1950).

Nature 167, 112 (1951) (see al so below).

Proc. Phys. Soc. London A 64, 458-464 (1951).

J. Opt. Soc. Am. $42,552-558$ (1952).

Zhur. Eksptl. i Teoret. Fiz. 27, $712-718$ (1954)

(SLA transl. RT-3363).

Vestnik Leningrad Univ. Ser. Fiz. i Khim.10, 135-142 (1955) (trans1.).

Soviet Phys. - Doklady 2, 167 169 (1957).

J. Opt. Soc. Am. 47, $156=162$ (1957).

Proc. Roy. Soc. London A 238, $473-488$ (1956-1957).

Thesis Harvard (1958).

Spectrochim. Acta 15, 349-359 (1959).

J. Opt. Soc. Am.51, 1058-1061 (1961).

Bull. soc. roy. sci. Liège 30, 287-299 (1961).

Geophysics Corporation of America Technical

Report 61-21-A; ASTIA Document 263459 (1961).

\section{K IV}

Astrophys. J. 114, 469-472 (1951).

quant. forb.

K VI

Astrophys. J. 114, 469-472 (1951).

$$
* * * * *
$$

$\mathrm{Kr} \quad \mathrm{I}$

Phys. and Chem. Solids 1, 284 (1957).

'Proceedings Fourth International Conference on Ionization Phenomena in Gases" 206-209

(ed. Nilsson, N. R., Nor th-Holland Publishing Co., Amsterdam, 1960).

Proc. Phys. Soc. London A 76, 833-843 (1960).

\section{$\mathrm{Kr} \| 11$}

Astrophys. J. 114, 469-472 (1951).

quant. forb.

\author{
hook, rel. \\ \& comm. \\ SCF \\ SCF \\ SCF \\ SCF \\ incomp l. \\ SCF \\ misc. \\ misc. \\ SCF \\ SCF \\ incomp l. \\ SCF \& estim. \\ emiss. \\ misc. \\ quant. \\ emiss. \\ CA \\ $\mathrm{CA}$ \\ CA
}

quant., rel. absorpt.

absorpt.

$$
* * * * *
$$

quant. forb. 
La \|

Boyarchuk, M. E. E Boyarchuk, A. A.

Akademi ia Nauk S.S.S.R. Krymskaia astrofizecheskaia observatorila. Izvestila 22, 234-256 (1960)

(transl.).

$x * x+\%$

Li I

*Kerschbaum, H.

* Kerschbaum, $\mathrm{H}$. Trumpy, B.

Trumpy, B.

*Hargreaves, J.

Trumpy, B.

Stevenson, A. F.

Trumpy, B.

Trumpy, B.

Filippov, A. N.

Filippor, A. N.

Filippov, A. N.

Fock, V. E Petrashen, M.

Key, J.

Bates, D. R. E Damgaard, A.

Sobolev, N. N.

Veselov, M. G.

Stephenson, G.

Vainshtein, L. A. E Yavorskii, B. M.

Vainshtein, L. A. E Yavorskii, B. M.

Hinnov, E. E Kohn, H.

Dronov, A. P., Sviridov, A. G. E Sobolev, N. N.

*Varsavsky, C. M.

Heavens, 0.S.

Naqvi, A. M.

Veselov, M. G.

Veselov, M. G.

Yutsis, A. P., Ushpalis, K. K., Kavetskis, V. I. E Levinson, I. B.
Sitzber, math-physik. Kl. bayer. Akad. Wiss. Muinchen 19-25 (1926) (see also below).

Ann. Physik 79, 465-488 (1926).

Z. Physik 44, 575-584 (1927). [See also ibid. 57, 787-796 (1929).]

z. Physik $50,228-233$ (1928) .

Proc. Cambridge Phil. Soc. 25, 75-96 (1929).

Z. Physik 27, 787-796 (1929)。

Proc. Roy. Soc. London A 128, 591-599 (1930).

z. Physik 61, 54-60 (1930) (see al so belowí).

Z. Physik 66,720 (1930).

Z. Physik 69, 526-547 (1931) (same as below).

Zhur. Eksptl。i Teoret. Fiz. 2, 24-41 (1932) (transl.) (same as above).

Trudy Gosudarst. Opt. Inst. Leningrad 8, 1-118 (1932) (transl.) (results same as above).

Physik. z. Sowjetunion 8, 547-561 (1935).

Thesis Utrecht (1937).

Phil. Trans. Roy. Soc. London. Ser. A. 242 $101-122$ (1949).

Zhur. Eksptl. i Teoret. Fiz. 19, 25-35 (1949) (transl.).

Zhur. Eksptl. i Teoret. Fiz. 19, 959-964 (1949) (ATS trans1.).

Nature 167, 156 (1951).

Doklady Akad. Nauk S.S.S.R. $87,919=922$ (1952).

Zhur. Ekspt1. i Teoret. Fiz。27, 712-718 (1954) (SLA transl. RT-3363).

J. Opt. Soc. Am. 47, 156-162 (1957).

Optika i Spektroskopiya 5, 490-499 (1958) (transl. Johns Hopkins APL Library Bulletin TG230-T159). Thesis Harvard (1958).

J. Opt. Soc. Am. 51, 1058-1061 (1961).

Geophysics Corporation of America Technical Report 6i-21-A; ASTIA Document 263459 (1961).

\section{Li II}

Zhur. Eksptl. i Teoret. Fiz. 19, 959-964 (1949) (ATS transi.).

Vestnik Leningrad Univ. Ser. Fiz. i Khim. No.8, $181-185$ (1953) (transl.).

Optika i Spektroskopiya 1, 601-605 (1956) (transl.). quant.

\author{
canal \\ canal \\ absorpt., rel. \\ quant. \\ SCF, rel. \\ absorpt., rel. \\ $\varepsilon$ comm. \\ quant. forb. \\ SCF \\ comm. \\ hook, rel. \\ hook, rel. \\ hook, rel. \\ \& comm. \\ SCF \\ emiss., rel. \\ SCF \\ incompl. \\ quant. \\ absorpt. \\ incompl. \\ SCF \& quant. \\ emiss. \\ emiss., rel. \\ quant. \\ CA \\ $C A$
}

quant.

quant. 
Mg |

Russell, H. N. Prokof'ev, V. K. Prokof'ev, V.K.

Rubenstein, P.J. Kersten, J.A. H.

Kersten, J.A.H. E Ornstein, L. S.

Schuttevaer, J. Wo, de Bont,

M. J. E van den Broek, Th. H.

Wilson, $0 . C$.

Biermann, L. E Trefftz, E.

Trefftz, E.

Trefftz, E.

Aller, L. H., Hazen, M., Doherty, L., Grant, G., Jugaku, J., Spiegel, E. E Waddell, J.

Allen, C. W.

Vainshtein, L. A.

Boldt, $G$.

Ostrovskii, Yu. 1., Penkin,

N. P. E Shabanova, L. N.

Ostrovksii, Yu. I., Penkin,

N. P. E Shabanova, L. N.

Varsavsky, C. M.

Addink, N. W. H.

Boyarchuk, M. E. E Boyarchuk, A. A.

Goldberg, L., Muiller, E. A. E Aller, L. H.

Brehm, B., Demtröder, W. E Osberghaus, 0 .

Demtröder, $W$.

Demtröder, $W$.

Russell, H. N.

Biermann, L.

Biermann, L.

Bi ermann, L.

Biermann, L. E Lübeck, K.

Aller, L. H., Hazen, M.g Doherty, L., Grant, Go, Jugaku, J., Spiegel, E. \& Waddell, J. Nagibina, 1. M.

Varsavsky, C. M. Boyarchuk, M. E. E Boyarchuk, A. A.
Astrophys. J. 78, 239-297 (1927).

Z. Physik 50, 701-715 (1928) (same as below).

Zhur. Ekspti. i Teoret. Fiz. I, $111=122$ (193i) (transl.) (same as above).

Phys. Rev. Letters 58, 1007 (1940).

Thesis Utrecht (194.1) (see below for results).

Physica 8, 1124-1136 (1941)。

Physica $10,544-552$ (1943).

Astrophys. J. 107, 126-150 (1948).

Z. Astrophys, 26, 213-239 (1949) (see also below).

Z. Astrophys. 26, 240-263 (1949) (see also below).

Z. Astrophys. 28, $67-78$ (1950).

Publications of the Observatory, Univ. of Michigan (Feb.19, 1954).

Monthly Notices Roy. Astron. Soc. 117, 622-628 (1957).

Optika i Spektroskopiya 3, 313-321 (1957) (transl.).

Z. Physik 150, 205-214 (1958).

Bul1. Acad. Sci. U.S.S.R. Phys. Ser. 22, 720-724 (1958) (results same as below).

Soviet Phys. - Doklady 3, $538=540$ (1958) (results same as above).

Thesis Harvard (1958).

Spectrochim. Acta 15, 349-359 (1959).

Akademi ia Nauk S.S.S.R. Krymskaia astrofizecheskaia observatorila. Izvestila 22, 234-256 (1960) (trans1.).

Astrophys. J. Suppl. Ser. 5, \#45, 1-137 (1960).

2. Naturforsch. 16a, 843 (1961) (see also below).

Thesis Bonn (1961) (see also below for results).

Z. Physik 166, 42-45 (1962).

\section{$\mathrm{Mg} \| \mathbb{1}$}

Astrophys. J. 78, 239-297 (1927).

Physik. Z. 44, 232-233 (1943) (see also below).

Z. Astrophys. 22, 157-164 (1943) (see also below). Nachr. Akad. Wiss. Göttingen, Math.-physik. Kl. $116-118(1946-1948)$ (see also below).

Z. Astrophys. 25, 325-339 (1948).

Publications of the Observatory, Univ. of Michigan (Feb 19, 1954).

Bull. Acad. Sci. U.S.S.R. Phys. Ser. 22, 678679 (1958).

Thes is Harvard (1958).

Akademila Nauk S.S.S.R. Krymskaia astrofizecheskaia observatorila. Izvestila 22, 234-256 (1960)

(transl.). estim.

estim.

estim.

quant., rel. emiss., rel. emiss., rel.

emiss., rel.

misc., rel.

SCF

SCF

SCF

misc., rel.

emiss. E CA

incompl. absorpt. hook

hook

quant.

emiss.

comm.

$\mathrm{CA}$

life

life

life

estim.

SCF

SCF

SCF

SCF

misc., rel.

emiss., rel.

quant.

comm.

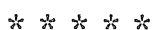


Mn I

Frerichs, R.

Seward, R. S.

Huldt, L. ELagerquist, A.

Huldt, L. E Lagerqvist, $A$.

Bell, G. D.

Nikonova, E. I. E Prokof'ev, V. K.

Ostrovskii, Yu. I., Parchevskii,

G. F。 E Penkin, N. P.

Allen, C. W. E Asaad, A. S.

Bell, G. D.

Hinnov, E. E Kohn, H.

Ostrovski i, Yu. I. EPenkin, N. P.

Dvornikova, 1. U. E Nagibina, I. M.

Nagibina, 1.M.

Addink, No W. H.

Bell, G. D., Davis, M. H.,

King, R. B. E Routly, P.M.

Allen, C. W.

Boyarchuk, M. E. E Boyarchuk, A. A.

Korolev, F.A. E Kvaratskheli, Iu. K.

Seward, R. S.

Wilson, 0. C.

Nagibina, I. M.

Boyarchuk, M. E. E Boyarchuk, A. A.

Nikonova, E. I. E Prokof'ev, v. K.
Ann. Physik 81, 807-845 (1926).

Phys. Rev. $37,344-361$ (1931).

Arkiv Fysik 5, $91-95$ (1951) (see also below).

J. Opt. Soc. Am. 42, 142 (1952) (results same as above).

Thesis California Institute of Technology (1956-

1957). [See Bell, G. Do, et al., Astrophys. J. 129, 437-440 (1959) for results.]

Optika i Spektroskopiya 1, 290-297 (1956) (transl.). hook, rel.

Optika i Spektroskopiya 1, 821-832 (1956) (transl.). incompl.

Monthly Notices Roy. Astron. Soc. 117, 36-49 (1957). emiss.

Astron.J.62, 7 (1957). [See Bell, G. D., et al., comm. Astrophys. J. 129, 437-440 (1959) for results.]

J. Opt. Soc. Am. 47, 156-162 (1957).

Optika i spektroskopiya 3, 193-201 (1957) (transl.). hook

Optika i Spektroskopiya 4, 421-429 (1958). incompl.

Optika i Spektroskopiya 4, 430-437 (1958) (transl.). incompl.

Spectrochim. Acta 15, 349-359 (1959).

Astrophys. J. 129, $437-440$ (1959).

Monthly Notices Roy. Astron. Soc. 121, 299-332 (1960).

Akademi ia Nauk S.S.S.R. Krymskaia astrofizecheskaia observatoriia. Izvestila 22, 234-256 (1960) (transl.).

Optics and Spectroscopy (U.S.S.R.) 10, 200-202 (1961).

Mn II

Phys. Rev. 37, 344-361 (1931).

Astrophys. Jo 107, 126-150 (1948).

Bul1. Acad. Sci. U.S.S.R. Phys. Ser. 22, 678= 679 (1958).

Akademi ia Nauk S.S.S.R. Krymskaia astrofizecheskaia observatoriia. Izvestila 22, 234-256 (1960) (trans1.).

$$
* x * * x
$$

Mo 1

Optika i Spektroskopiya 1, 290-297 (1956)

(transl.).

$$
* * * * *
$$

N

J. Geophys. Research 55, 143-151 (1950).

Astrophys. J. 111, 580-581 (1950).

Astrophys. J. 115, 506-508 (1952).

Z. Physik 143, 77-92 (1955).

"The Airglow \& the Aurorae,":324-327 (ed. Armstrong \& Dalgarno, Pergamon Press, New York, 1956).

Thes is Harvard (1958). emiss., rel. misc., rel. emiss., rel.

comm。

hook, rel.

quant. quant. forb., rel. quant. forb. emiss. quant. forb. quant. 
Bates, D. R.

Goldberg, L., Muiller, E. A. E Aller, L. H.

Doherty, L. R.

Naqvi, A. M.

Richter, J.

*Stevenson, A. F。

*Condon, E. U.

Garstang, R. H.

Vainshtein, L. A. E Yavorskii, B. M.

Yamanouchi, T. E Horie, $H$.

Mandel'shtam, S. L. E Sukhodrev, N. K.

Yilmaz, H.

Bolotin, A. B., Levinson, 1. B.

E Levin, L. I .

McCarrol 1, R. J. E Wakely, P. G.

Mastrup, F。 E Wiese, W.

*Varsavsky, C. M.

Aller, L. H. E Jugaku, J.

Bates, D.R.

Gaustad, J.E. E Spitzer, Jr., L.

Jugaku, J., Sargent, L.W.

\& Greenstein, J. L.

Naqvi, A. M.

Aller, L. H.

Vainshtein, L. A. E Yavorskii, B. M.

Bolotin, A. B. E Yutsis, A. P.

Mandel 'shtam, S. L. E Sukhodrev, N. K.

Gaustad, J. E. E Spitzer, Jr., L.

Aller, L. H.

Bolotin, A. B. E Yutsis, A. P.
"Physics of the Upper Atmosphere," 300-302

(ed. Ratcliffe, J. A., Academic Press, New York, 1960).

Astrophys. J. Supp 1. Ser. 5, \#45, 1-137 (1960).

Thesis Michigan (1961).

Geophysics Corporation of America Technical Report 61-21-A; ASTIA Document 263459 (1961).

Z. Astrophys. 51, 177-186 (1961).

\section{$N \|$}

Proc. Roy. Soc. London A 137, 298-325 (1932).

Astrophys. J. 79, 217-234 (1934).

Monthly Notices Roy. Astron. Soc. 111, 115-124 (1951).

Doklady Akad. Nauk S.S.S.R. 87, 919-922 (1952) (transi.).

J. Phys. Soc. Japan I, 52-56 (1952).

Zhur. Eksptl. i Teoret. Fiz. 24, 701-707 (1953).

Phys. Rev。 100, 1148-1153 (1955).

Soviet Phys. = JETP 2, $391-395$ (1956).

"The Airglow and the Aurorae," 337-339 (ed.

Armstrong \& Dalgarno, Pergamon Press, New York, 1956).

Z. Astrophys. 44, 259-279 (1958).

Thes is Harvard (1958).

Astrophys. J. Supp 1. Ser. 4, \#38, 109-156 (1959).

"Physics of the Upper Atmosphere" 300-302 (ed.

Ratcl iffe, J.A., Academic Press, New York, 1960).

Astrophys. J. 134, 771-776 (1961).

Astrophys. J. 134, 783-796 (1961).

Geophysics Corporation of America Technical Report 61-21-A; ASTIA Document 263459 (1961).

\section{$N\|\|$}

Astrophys. J. 27, 135-165 (1943).

Doklady Akad. Nauk S.S.S.R. 87, 919-922 (1952) (transl.).

Zhur. Eksptl. i Teoret. Fiz. 24, 537-543 (1953) (transl.).

Zhur. Eksptl. i Teoret. Fiz. 24, 701-707 (1953) (transl.).

Astrophys. J. 134, 771-776 (1961).

N IV

Astrophys. J. 97, 135-165 (1943).

Zhur. Eksptl. i Teoret. Fiz。 24, 537-543 (1953)

(transl.). comm. forb.

CA

emiss.

CA \& comm.

forb.

emiss.

quant. forb. quant. forb. quant. forb.

incomp 1 .

quant. forb. incomp 1 .

quant. forb. quant.

SCF

emiss. quant.

CA

comm. forb.

estim。

CA

comm. forb.

quant. incompl.

SCF

incompl .

estim.

quant. SCF 
$\mathrm{Na} I$

Ladenburg, R. E Minkowski, R. Minkowski, R.

Minkowski, R.

Thomas, W.

Tolman, R. C.

Bartels, $H$.

Ellett, $A$.

Gerlach, W. E Schuitz, W.

Harrison, G. R.

Hoyt, F. C.

Trumpy, B.

Kerschbaum, $H$.

Kerschbaum, $\mathrm{H}$.

Sugiura, $Y$.

Trumpy, B.

Trumpy, B.

Bl eeker, C. E.

Ladenburg, $R$.

Ladenburg, R. E Minkowski, R.

Ornstein, L. S. E van der Held, E. F. M.

Filippov, A. E Prokof"ev, V. K. Hupfeld, $H$.

Prokof"ev, V. K.

Prokof'ev, V. K.

Weiss, C.

Ladenburg, R. \& Thiele, E.

Stevenson, A. F.

Trumpy, B.

Berry, N. E. E Rollefson, G. K. Ladenburg, R. E Thiele, E。

Prokof" ev, V. K.

Weingeroff, M.

Zehden, W.

Duschinsky, $F$.

Filippov, A. N.

van der Held, E. F. M.

van der Held, E. F. M. E

Ornstein, L. S.

Ornstein, L. S. E Key, J.

Zehden, W.

Fock, $V$.

Ornstein, L. S. \& Key, J。

Fock, V. E Petrashen, M.

Minkowski, R. \& Weber= Schäfer, M.

Minkowski, R., Mi̊ller, H. G. E Weber-Schåfer, M.

Righini, G.

Key, J.

Rozhestvenskii, D. S. E Penkin, N. P.

Rozhestvenskii, D. S. E Penkin, N. P.

Biermann, L.

Biermann, L.
Z. Physik 6, 153-164 (1921) (see also below). Thesis Breslau (1921) (see below for results). Ann. Physik 66, 206-226 (1921).

Z. Physik 24, 169-196 (1924).

Phys. Rev. 23, 693 $=709$ (1924).

z. Physik 32, $415-438$ (1925).

J. Opt. Soc. Am. 10, 427-437 (1925).

Physik. Z. 26, 33-35 (1925)。

Phys. Rev。 $25,768=782$ (1925).

Phys. Rev. 26, 749-760 (1925)。

Z. Physik 34, 715-721 (1925).

Ann. Physik 79, 465-488 (1926).

Sitzber. math.mphsik. Kl bayer. Akad. Wiss. Muinchen, 19a-25 (1926) (see al so above).

Phil. Mag. 4, 495-504 (1927)。

z. Physik $42,327=328$ (1927).

$\mathrm{Kgl}$. Norske Videnskab. Selskabs, Skrifter 1 , I-80 (1927).

Thesis Utrecht (1928).

Z. Physik 48, 15-25 (1928).

Ann. Physik 87, 298-306 (1928).

Ann. Physik 85, 953-960 (1928).

Z. Physik 56, 458-476 (1929).

z. Physik 54, 484-497 (1929).

Z. Physik 57, 387-393 (1929). [Same as Zhur.

Eksptl. i Teoret. Fiz. 1, 123-127 (1931).]

z. Physik 58, 255-267 (1929).

Ann. Physik 1, 565-612 (1929).

Z. physik. Chem. B Z, 161-187 (1930).

Proc. Roy. Soc. London A 128, $591-599$ (1930).

Z. Physik $61,54-60(1930)$.

Phys. Rev. 38, 1599-1611 (1931).

Z. Physik 72, 697-699 (1931).

Zhur. Eksptl. i Teoret. Fiz. 1, 123-127 (1931)

(transl.). [Same as Z. Physik 27, 387-393 (1929).]

Z. Physik 67, 679-698 (1931).

Naturwi ssenschaften 19, 826-827 (1931).

Z. Physik 78, 586-602 (1932).

Zhur. Eksptl. i Teoret. Fiz. 2, 24-41 (1932) (transl.).

Thesis Utrecht (1932) (trans1.) (see below for results).

Z. Physik Z7, 459-477 (1932).

Z. Physik 85, 565-567 (1933).

Z. Physik 86, 555-582 (1933).

Z. Physik $89,744-749$ (1934).

Physica $1,945=952$ (1934).

Physik. Z. Sowjetunion 8, 547-561 (1935).

Z. Physik 94, 172-175 (1935).

Z. Physik 94, 145-171 (1935)。

Z. Astrophys. 10, 344-352 (1935).

Thes is Utrecht (1937).

J. Phys. (U.S.S.R.) 5, 319-337 (1941).

Izvest. Akad. Nauk S.S.S.R. Ser. Fiz. 5, 97-101 (1941) (transi.).

Z. Astrophys. 22, 157-164 (1943) (see al so below). Physik. Z. 44, 232-233 (1943). [See al so Nachr. Akad. Wiss. Göttingen Math.=physik. Kl. 116-118 $(1946)$. misc。

misc.

misc.

estim.

comm.

quant.

misc.

comm.

absorpt., rel. quant o rel. absorpt., rel.

canal

canal

quant.

comm.

absorpt.

emiss., rel.

comm.

comm.

emiss.

hook, rel.

life

hook forb.,

rel.

quant.

emiss., rel.

comm.

quant. forb.

SCF

emiss., rel.

comm.

hook forb.,

rel.

misc.

absorpt.

life

hook, rel.

emiss.

emiss.

emiss., rel. absorpt.

comm.

emiss., rel.

SCF

comm.

emiss.

estim. emiss., rel. hook, rel. E comm. hook, rel.

SCF

SCF 
Paul, W.

Kvater, G. S.

Biermann, L.

Dupuy, G.

Kvater, G. S.

Biermann, L. E Lübeck, K.

Bates, D. R. E Damgaard, A.

Sobolev, N. N.

Seaton, M. J.

Stephenson, G.

Stephenson, G.

Vainshtein, L. A. E Yavorskii, B. M.

Petrashen, M. I. E Abarenkov, I. V.

Vainshtein, L. A. \& Yavorski i, B. M.

Yanoukh, $F$ 。

Anderson, E. M., Buska, Z. A., Grinberg, R. O. E Saulgozha, A. K.

Bersuker, 1. B.

Hinnov, E. E Kohn, H.

Vainshtein, L. A.

Bersuker, I. B.

Descoubes, J.P. E Pebay=

Peyroula, J. C.

Dronov, A. P., Sviridov, A. G. E Sobolev, N. N.

Krüger, H. E Scheffler, K.

Varsavsky, C. M.

Addink, N. W. H.

Archambaul t, $Y_{0}$, Descoubes, J. P., Priou, M., Omont, A. E Pebay-Peyroula, J. C.

Goldberg, L., Mioll ler, E. A. E Aller, L. H.

Brehm, B., Demtro̊der, W. E Osberghaus, 0 .

Demtröder, $W$.

Heavens, 0. S.

Houziaux, L. E Sadoine, M. P. Naqvi, A. M.

Ostrovskii, Yu. I. EPenkin, N. P.

Demtro̊der, $W$.

Petersen, R.
Z. Physik 124, 121-128 (1944).

Izvest. Akad. Nauk S.S.S.R. Ser. Fiz. 2 236-237 (1945) (transl.).

Nachr. Akad. Wiss. Go̊ttingen Math.-physik. K1. 116-118 (1946). [See also Biermann, L. E Luibeck, K., Z. Astrophys. 25, 325-339 (1948).]

Compt. rend. 222, 654-656 (1946) (transl.).

Vestnik Leningrad Univ. Ser. Fiz. i Khim. No.2, $135-141$ (1947) (trans1.).

Z. Astrophys. 25, 325-339 (1948).

Phil. Trans. Roy. Soc. London. Ser. A. 242 , $101-122$ (1949).

Zhur. Eksptl. i Teoret. Fiz, 19, 25=35 (1949) (transl.).

Proc. Roy. Soc. London A 208, $418-430$ (1951).

Nature 167, 112 (1951) (see al so below).

Proc. Phys. Soc. London A 64, 458-464 (1951).

Doklady Akad. Nauk S.S.S.R. 87, 919-922 (1952) (transl.).

Vestnik Leningrad Univ. Ser. Fiz. i Khim. No.9, $141-148$ (1954)

Zhur. Eksptl. i Teoret. Fiz. 27, $712=718$ (1954) (SLA trans1. RT-3363).

Vestnik Leningrad Univ. Ser. Fiz. i Khim. No. 10, $135-142$ (1955) (transl.).

Vestnik Leningrad Univ. Ser. Fiz. i Khim. No. 4, 27-31 (1956) (transi.).

Soviet Phys. - Doklady 2, 167-169 (1957).

J. Opt. Soc. Am. 47, 156-162 (1957).

Optika i Spektroskopiya 3, 313-321 (1957) (transl.).

Bull. Acad. Sci. U.S.S.R. Phys. Ser. 22, 743746 (1958).

Compt. rend. 247, 2330 $=2332$ (1958) (transi.).

Optika i Spektroskopiya 5, 490-499 (1958) (transi. Johns Hopkins APL Library Bulletin TG230-T159).

J. phys. radium 19, 854-857 (1958).

Thes is Harvard (1958).

Spectrochim. Acta 15, 349-359 (1959).

J. phys. radium 21, 677-679 (1959) (trans1.).

Astrophys. J. Suppl. Ser. 5, \#45, 1-137 (1960).

Z. Naturforsch.16a, 843 (1961). [See also Demtröder, W., Thesis Bonn (1961) and Z. Physik $166,42-45$ (1962).]

Thesis Bonn (1961). [See Z. Physik 166, 42-45 (1962) for results.]

J. Opt. Soc. Am. 51, 1058=1061 (1961).

Bull. soc. roy, sci. Liège $30,287=299$ (1961).

Geophysics Corporation of America Technical Report 61-21-A; ASTIA Document 263459 (1961).

Optics and Spectroscopy (U.S.S.R.) 11, I-5 (196i).

Z. Physik 166, 42-45 (1962).

Na $\|$

Phys. and Chem. Solids 1, 284 (1957).

$* * * * *$ misc。

incompl.

SCF

comm.

incomp l.

SCF

CA \& SCF

incompl.

comm.

misc.

misc.

incompl.

incompl.

SCF \& quant.

incomp l.

incompl.

SCF \& estim.

emiss.

incomp 1 .

SCF

misc.

emiss., rel.

misc。

quant.

emiss.

misc.

CA

life

life

CA

CA

$\mathrm{CA}$

hook

life

quant., rel. 
$\mathrm{Ne} I$

Ladenburg, R., Kopfermann, $H_{\text {. }}$ E Carst, A.

Kopfermann, H. E Ladenburg, R. Kopfermann, $H$. \& Ladenburg, R. Ladenburg, $R$.

Schütz, W.

Griffiths, J.H.E.

Ladenburg, R. E Levy, S.

Shortley, G. H.

Garbuny, M.

Ufford, C. W.

Phelps, A. V.

Petersen, R.

*Varsavsky, C.M.

Gold, A. E Knox, R. S.

Phelps, A. V.

Osherovich, A. L. E Petelin, G. M.

Doherty, L. R.

Frish, S. Z. E Bochkova, O. P.

Garstang, R. H.

Garstang, R. H.

Traving, G.

Aller, L. H., Elste, G. $E$ Jugaku, J.

Garstang, R. H.

Garstang, R. H.

obi, s.

Garstang, R. H.

Bolotin, A. B., Levinson, 1. B. \& Levin, L. I.

Veselov, M. G.

Veselov, M. G.
Sitzber. deut. Akad. Wiss. Berlin, Kl. Math., Phys. u Tech. No. $21,255-273$ (1926).

Z. Physik 48, 51-61 (1928) (see al so below).

z. Physik $65,167-188$ (1930) (see al so below). Rev. Mod. Phys. 5, 243-256 (1933).

Ann. Physik 18, 705-720 (1933).

Proc. Roy. Soc. London A 143, 588-604 (1934).

Z. Physik 88, 461-468 (1934).

Phys. Rev. 47, 295-300 (1935).

Z. Physik 107, 362-368 (1937)。

Astrophys. J. 85, 249-250 (1937).

Phys. Rev. 100, 1230 (1955).

Phys. and Chem. Solids I, 284 (1957).

Thesis Harvard (1958).

Phys. Rev. $113,834-839$ (1959).

Phys. Rev. 114, 1011-1025 (1959).

Soviet Phys。 - Doklady 4, 1289-1291 (1960).

Thesis Michigan (1961).

Vestnik Leningrad Univ. Ser. Fiz. i Khim. No. 16, 40-58 (1961) (trans1.).

$\mathrm{Ne}$ II

Monthly Notices Roy. Astron. Soc. 110, 612-614 (1950).

Monthly Notices Roy. Astron. Soc. 114, 118=133 (1954).

Z. Astrophys. 36, 1-41 (1955).

Astrophys. J. Supp 1. Ser. 3, \#25, 1-35 (1957).

Ne \|\|

Monthly Notices Roy. Astron. Soc. 111, 115-124 (1951).

Ne IV

Monthly Notices Roy. Astron. Soc, 120, 201-203

(1960).

Ne $V$

Publ. Astron. Soc. Japan 2, 150-155 (1950).

Monthly Notices Roy. Astron. Soc. 111, 115-124 (1951).

Soviet Phys。 - JETP 2, 391-395 (1956).

Ne VII

Zhur. Ekspt1。i Teoret. Fiz, 19, 959-964 (1949) (ATS transl.).

Vestnik Leningrad Univ. Ser. Fiz. i Khim. No. 8, $181-185$ (1953) (transl.). hook

hook

hook

hook, hook, rel. \& comm.

misc.

life

emiss.

quant. E comm.

emiss., rel.

quant.

life forb.

quant., rel.

quant.

SCF

comm., rel.

life

emiss.

incompl.

CA \& SCF

quant., rel. \& $C A$

$\mathrm{CA}$

$\mathrm{CA}$

quant. forb.

quant. forb.

quant. forb.

rel.

quant. forb.

quant.

quant.

quant. 
Ne VIII

Veselov, M. G.

Veselov, M. G.

Veselov, M. G.

Bouma, $T$.

Ornstein, L. S. E Bouma, T.

van Driel, $H$.

King, R. B.

Wilson, 0 . C.

Estabrook, F. B.

Estabrook, F. B.

Parchevski i, G. F. E Penkin, N. P.

Allen, C. W. E Asaad, A. S. Hinnov, E. E Kohn, H.

Varsavsky, C. M.

Addink, N. W. H.

Allen, C. W.

Boyarchuk, M. E. E Boyarchuk, A. A.

Mitrofanova, L. A.

Ornstein, L. S. E Bouma, T.

Aller, L. Ho, Hazen, M.,

Doherty, L., Grant, G., Jugaku, J., Spiegel, E. E Waddell, J.

Garstang, R. H.

Boyarchuk, M. E. E Boyarchuk, A. A.

Groth, H. G.

Garstang, R. H.
Zhur. Eksptl. i Teoret. Fiz. 19, 959-964 (1949)

(ATS transl.).

$\mathrm{Ne} I X$

Zhur. Eksptl. i Teoret. Fiz. 19, 959-964. (1949)

(ATS transl.).

Vestnik Leningrad Univ. Ser. Fiz. i Khim. No. 8, 181-185 (1953) (transl.).

$$
* * * * *
$$

Ni I

Thesis Utrecht (1930) (see below for results).

Phys. Rev. 36, 679-693 (1930).

Thesis Utrecht (1935).

Astrophys. J. 108, 87-91 (1948).

Astrophys. J. $107,126-150$ (1948).

Thesis California Institute of Technology (1950) (see below for results).

Astrophys. J. 113, 684-689 (1951).

Bull. Acad. Sci. U.S.S.R. Phys. Ser. 19, 4-5 (1955).

Monthly Notices Roy. Astron. Soc. 117, 36-49 (1957).

J. Opt. Soc.Am. 47, 156-162 (1957).

Thes is Harvard (1958).

Spectrochim. Acta 15, 349-359 (1959).

Monthly Notices Roy. Astron. Soc. 121, 299-332 (1960).

Akademi ia Nauk S.S.S.R. Krymskaia astrofizecheskaia observatoriia. Izvestila 22, 234-256 (1960) (transl.).

Pulkovo. Astronomi cheskaia observatori ia. Izvestila 21, 185-189 (1960) (transl.).

\section{Ni 11}

Phys. Rev. 36, 679-693 (1930).

Publications of the Observatory, Univ. of Michigan (Feb. 19, 1954).

Monthly Notices Roy. Astron. Soc. 118, 234-240 (1958).

Akademila Nauk S.S.S.R. Krymskaia astrofizecheskaia observatoriia. Izvestila 22, 234-256 (1960). (transl.).

z. Astrophys. 51, 231-285 (1961).

$\mathrm{Ni}\|\|$

Monthly Notices Roy. Astron. Soc. $118,234-240$ (1958). quant.

quant.

quant.

emiss., rel. emiss., rel. emiss., rel. absorpt., rel. misc., rel. absorpt.

absorpt. comm.

emiss. emiss. quant. emiss. comm. \& CA comm.

emiss., rel.

emiss., rel. misc., rel.

quant. forb. comm.

misc。

quant. forb. 
Ni X $\|$

Edlén, $B$. Huang, $K$.

Edlén, $B$. Huang, $K$.

Edlén, B. Huang, $K$.

Edlén, B. Huang, K.
Z. Astrophys. 22, 30-64 (1942). Astrophys. J. 101, 187-195 (1945).

$\mathrm{Ni} X\|\|$

Z. Astrophys. 22, 30-64 (1942). Astrophys. J. 101, 187-195 (1945).

Ni XV

Z. Astrophys. 22, 30-64 (1942). Astrophys.J.101, 187-195 (1945).

Ni XVI

Z. Astrophys. 22, 30-64 (1942). Astrophys. J. 101, 187-195 (1945).

$$
* * * * *
$$

\section{1}

Astrophys. J. 78, 239-297 (1927).

Astrophys. J. 79, 217-234 (1934).

Rev. Mod.Phys. 20, 109-112 (1948).

Can. J. Research 27A, 213-217 (1949).

Nature 166, 656-657 (1950).

J. Geophys. Research 55, 143-151 (1950).

Monthly Notices Roy. Astron. Soc. 111,115 124 (1951).

Proc. Phys. Soc. London A 65, 219-226 (1952).

[See al so Emeleus, K. G., et al., Nature 166, 656-657 (1950).]

J. Phys. Soc. Japan Z, 52-56 (1952).

"Proceedings of the Conference on Auroral Physics" No. 30, 221-241 (1954).

Z. Physik 138, 613-622 (1954).

Phys. Rev. 99, 1846-1850 (1955).

J. Atmospheric and Terrest. Phys. 7, 247-253

(1955). [See also Omholt, A., Geofys.

Publikasjoner Norske Videnskaps-Akad. i Oslo 21, 1-38 (1959).]

"The Airglow and the Aurorae" $324=327$ (ed.

Armstrong and Dalgarno, Pergamon Press, New York, 1956).

Omhol t, A.

Omhol t, A.

Vainshtein, L. A.

Liszka, L. E Ni ewodni czanski, H.

*Varsavsky, C. M. Omhol t, A.

Bates, D. R.
J. Atmospheric and Terrest. Phys.9, 28-35 (1956).

"The Airglow and the Aurorae" 178-182 (ed.

Armstrong and Dalgarno, Pergamon Press, New York, 1956). [See also Geofys. Publikasjoner Norske Videnskaps-Akad. i Oslo 21, 1-38 (1959).]

Optika i spektroskopiya $3, \frac{21}{313-321}$ (1957) (transl.). Acta Phys. Polon. 17, 345 $=351$ (1958).

Thesis Harvard (1958).

Geofys. Publikasjoner Norske Videnskaps-Akad. i Oslo 21, 1-38 (1959).

"Physics of the Upper Atmosphere" 300-302 (ed. Ratcl iffe, J.A., Academic Press, New York, 1960). quant. forb. misc. forb.

quant. forb. misc. forb.

quant. forb. misc. forb.

quant. forb. misc. forb.

estim. quant. forb. estim. quant.

life forb.

quant. quant. forb.

life forb. E comm.

quant. forb. life. forb.

emiss.

SCF

life forb.

quant. forb.

$C A \& S C F$

life forb. E comm.

incompl. emiss. forb., rel.

quant. misc. \&life forb. comm. forb. 
Goldberg, L., Mi̊ller, E. A. \& Aller, L. H.

Omholt, A.

Stoffregen, W. E Derblom, $H$. Doherty, L. R.

Garstang, R. H. J.B.

Foster, E. W.
Naqvi, A. M.

Wiese, W. L. E Shumaker, Jr.,

Astrophys. d. Supp 1. Ser. 5, 估5, 1-137 (1960).

Planetary and Space Science 2, 246-248 (1960).

Nature 185, 28-29 (1960) (see also above).

Thesis Michigan (1961).

Proc. Cambridge Phil. Soc. 57, 115-120 (1961).

Geophysics Corporation of America Technical Report 61-21=A; ASTIA Document 263459 (1961). J. Opt. Soc. Am. 51, 937-942 (1961).

Proc. Phys. Soc. London A 79, 94-104 (1962).

$0 \|$

*Russell, H. N. Rubinowicz, A.

*Condon, E. U.

Aller, L. H. E Menzel, D. H. Bates, D. R. \& Damgaard, A.

Aller, L. H., Ufford, C. W. E van Vleck, J.H.

Garstang, R. H.

Yamanouchi, T. E Horie, H. Garstang, R. H.

Traving, G.

Garstang, R. H.

Aller, L. H., Elste, G. E Jugaku, J.

Naqvi, A. M. E Talwar, S. P.

Seaton, M. J. E Osterbrock, D. E.

Mastrup, F. E Wiese, W.

*Varsavsky, C.M.

Aller, L. H. E Jugaku, J.

Bates, D. R.

Naqvi, A. M.
Astrophys. J. 78, 239-297 (1927).

2. Physik 65, 662-676 (1930).

Astrophys. J. 79, 217-234 (1934).

Astrophys. J. 102, 239-263 (1945).

Astrophys. J. 107, 383-385 (1948).

Astrophys. J. 109, $42-52$ (1949).

Astrophys. J. 115, 506-508 (1952)

J. Phys. Soc. Japan Z, 52-56 (1952).

Monthly Notices Roy. Astron. Soc. 114, 118-133 (1954).

2. Astrophys. 36, 1-41 (1955).

"The Airglow and the Aurorae" 324-327 (ed.

Armstrong \& Dalgarno, Pergamon Press, New York, 1956).

Astrophys. J. Suppl. Ser. 3, \#25, 1-35 (1957).

Monthly Notices Roy. Astron. Soc. 117, 463-471 (1957).

Astrophys.J. 125, 66-83 (1957).

Z. Astrophys. 44, 259-279 (1958).

Thesis Harvard (1958).

Astrophys. J. Suppl. Ser. 4, \#38, 109-156 (1959).

iphysics of the Upper Atmosphereil, 300-302 (ed. Ratcliffe, J.A., Academic Press, New York, 1960).

Geophysics Corporation of America Technical Report 61-21-A; ASTIA Document 263459 (1961).

\section{0\|\|}

Proc. Roy. Soc. London A 137, 298-325 (1932).

Astrophys. J. 79, 217-234 (1934).

Astrophys. J. 94, 436-448 (1941).

Astrophys. J. 97, 135-165 (1943).

Publ. Astron. Soc. Japan 2, 150-155 (1950).

Monthly Notices Roy. Astron. Soc. 111, 115=124 (1951).

J. Phys. Soc. Japan Z 52-56 (1952).

Phys. Rev. 100, 1148-1153 (1955).

Soviet Phys。 JETP 2, 391-395 (1956).

"Physics of the Upper Atmosphere", 300-302 (ed. Ratcliffe, J.A., Academic Press, New York, 1960).
CA

1 ife forb. life forb. emiss.

quant. \& quant. forb. $\mathrm{CA}$

emiss.

eniss.

estim. quant., rel. quant. forb. quant.

SCF

quant. forb.

quant. forb. quant. forb. quant., rel. \& CA

$\mathrm{CA}$

quant. forb.

CA

quant. forb.

quant. forb.

emiss.

quant.

CA

comm. forb.

CA \& comm. forb.

quant. forb quant. forb. quant. quant. quant. forb., rel. quant. forb.

quant. forb. quant. forb. quant.

comm. forb. 


\section{o IV}

Aller, L. H.

Bolotin, A. B. E Yutsis, A. P.

Aller, L. H.

Bolotin, A. B. E Yutsis, A. P.

Jugaku, J., Sargent, L.W.

E Greenstein, J.L.

Mrozowski, S。

Gerjuoy, E.

Jenkins, F. A. E Mrozowski, S.

Engler, H. D.

Allen, C. W.

Allen, C. W. E Asaad, A. S.

Khokhlov, M. Z.

Bell, G. D. E King, R. B.

Khokhlov, M. Z.

Khokhlov, M. Z.

Bell, G. D. E King, R. B. Helliwell, T.M.

Bleeker, C. E.

Prokof'ev, V. K.

Stevenson, A. F. Prokof'ev, V. K.

Ornstein, L. S. E Key, J. Prokof'ev, V. K. E Shtandel, G.

Heierman, J.H.
Astrophys. J.97, 135-165 (1943).

Zhur. Eksptl. i Teoret. Fiz. 24, 537-543 (1953) (transl.).

\section{o v}

Astrophys. J. 97, 135-165 (1943).

Zhur. Eksptl. i Teoret. Fiz。24, 537-543 (1953) (transl.).

$$
* * * * *
$$

$P \|$

Astrophys. J. 134, 783-796 (1961).

$$
* * * * *
$$

$\mathrm{Pb} \mid$

Phys. Rev. 58, 1086-1093 (1940).

Phys. Rev. 60, 233-240 (1941).

Phys. Rev. 60, 225-233 (1941).

Z. Physik 144, 343-353 (1956).

Monthly Notices Roy. Astron. Soc. 117, 622-628 (1957).

Monthly Notices Roy. Astron. Soc, $117,36=49$ (1957) (see also above).

Akademi ia Nauk S.S.S.R. Krymskaia astrofizecheskaia observatoriia. Izvestila 21, 84-102 (1959) (transi.).

Astron. J. $65,483(1960)$. [See al so Astrophys. J. $133,718-722(1960)$.]

Akademila Nauk S.S.S.R. Krymskaia astrofizecheskaia observatoriia. Izvestila 22, 118-127 (1960) (trans1.).

Akademi ia Nauk S.S.S.R. Krymskaia astrofizecheskaia observatoriia. Izvestila 22, 128-133 (1960) (trans1.).

Astrophys. J. 133, 718-722 (1961).

Astrophys. J. 133, 566-571 (1961).

$$
* * * * *
$$

$\mathrm{Rb}$

Thesis Utrecht (1928).

Z. Physik 57, $387-393$ (1929). [Same as Zhur. Ekspt1. i Teoret. Fiz. 1, 123-127 (1931).]

Proc. Roy. Soc. London A 128, 591-599 (1930).

Zhur. Ekspt1.i Teoret. Fiz。 1, 123-127 (1931) (transl.). [Same as Z. Physik 57, 387-393 (1929).]

Physica 1, 945-952 (1934).

Zhur. Eksptl. i Teoret. Fiz. 4, 359-367 (1934) (transi.).

Thesis Utrecht (1937).
CA

quant. SCF

quant. SCF

emiss. forb., rel.

quant. forb. emiss. forb., rel.

absorpt. comm.

emiss.

emiss.

absorpt.

absorpt. $\varepsilon$ emiss.

incompl.

absorpt. quant.

\footnotetext{
emiss., rel. hook forb. rel. quant. forb. hook forb. rel.

comm., rel. hook, rel.

emiss.
} 
Key, J.

Stephenson, G.

Stephenson, $G$.

Meyer-Berkhout, U.

Yanoukh, $F$.

Gol'dberg, G. I.

Heavens, 0. So

Naqvi, A. M.

Petersen, R.

Edlén, B.

Goldberg, L., Müller, E. A. \& Aller, L. H.

Bowen, I. S.

Osterbrock, D. E.

Goldberg, L., Mưll ler, E. A.

\& Aller, L. H.

Condon, E. U.

Garstang, R. H.

Garstang, R. H.

Naqvi, A. M. E Talwar, S. P.

Aller, L. H. E Jugaku, J.

Gaustad, J.E. E Spitzer, Jr., L.

Gaustad, J. E. E Spitzer, Jr., L.

\section{S IV}

Gaustad, J.E. E Spitzer, Jr., L.

Z. Physik 141, 185-197 (1955). $135-142$ (1955) (transl.). 20, 126-137 (1956) (transl.).

$\mathrm{Rb} \|$

$* * * * *$

Rn II

Phys. Rev. 65, 248 (1948).

$* * * * *$

Ru I

$* * * * *$

S I

Astrophys. J. 114, 469-472 (1951).

\section{$S \|$}

Astrophys. J. 29, 217-234 (1934).

$$
\text { (1954). }
$$

(1957).

Astrophys. J. 134, 771-776 (1961).

$S\|\|$

Astrophys. J. 134, 771-776 (1961).
Astrophys. J. 134, 771-776 (1961).

Thesis Utrecht (1937).

Nature 167, 112 (1951) (see also below).

Proc. Phys. Soc. London A 64, 458-464 (1951).

Vestnik Leningrad Univ. Ser. Fiz. i Khim.10,

Pulkovo. Astronomicheskaia observatorila. Izvestila

J. Opt. Soc. Am. 51, 1058-1061 (1961).

Geophysics Corporation of America Technical

Report 61-21-A; ASTIA Document 263459 (1961).

Phys. E Chem. Solids 1, 284 (1957).

Astrophys. J. Suppl. Ser. 5, \#45, 1-137 (1960).

Rev. Mod. Phys. 20, 109-112 (1948).

Astrophys. J. Suppl. Ser. 5, \#45, 1-137 (1960).

Astrophys. J. 115, 506-508 (1952).

Monthly Notices Roy. Astron. Soc. 114, 118-133

Monthly Notices Roy. Astron. Soc. 117, 463-471

Astrophys. J. Suppl. Ser. 4, \#38, 109-156 (1959).

estim.

estim.

emiss., rel.

misc.

misc。

misc。

incompl.

hook, rel.

CA

CA

quant., rel.

quant. forb.

estim. forb. quant. forb. CA

quant. forb. quant. forb. quant., rel. \& $C A$ quant. forb.

CA

estim.

$* * * * *$ 
Sc 1

Ostrovskii, Yu. I.EPenkin, N.P.

Mitrofanova, L. A.

Allen, C. W.

Goldberg, L., Müller, E. A. E. Aller, L. H.

Wilson, 0. C.

Aller, L. H., Hazen, M., Doherty, L., Grant, G., Jugaku, J., Spiegel, E. E Waddell, J.

Mi trofanova, L. A.

Boyarchuk, M. E. E Boyarchuk, A. A.

Groth, H. G.

Boyarchuk, M. E. E Boyarchuk, A. A.

Russel 1, H. N.

Allen, C. W.

Allen, C. W. E Asaad, A. S.

Varsavsky, C. M.

Addink, N. W. H.

Hey, P.

Boyarchuk, M. E. E Boyarchuk, A. A.

Goldberg, L., Muiller, E. A. \& Aller, L. H.

Vidale, G. L.

Russel1, H. N.

Biermann, L.

Bates, D. R. E Damgaard, A. Biermann, L. E Lübeck, K.

Aller, L. H., Hazen, M., Doherty, L., Grant, G., Jugaku, J., Spiegel, E. \& Waddell, J.

\author{
Optika i Spektroskopiya 3, 391-393 (1957) (transl.). hook, rel. \\ Pulkovo. Astronomicheskaia observatorila. Izvestila emiss., rel. \\ 21, \#162, 159-161 (1958) (transi.). \\ Monthly Notices Roy. Astron. Soc. 121, 299-332 (1960)。comm.E CA \\ Astrophys. J. Supp 1. Ser. 5, \#45, T-137 (1960). \\ CA
}

Sc $\|$

Astrophys. J. 107, 126-150 (1948).

Publications of the Observatory, Univ. of

Michigan (Feb. 19, 1954).

misc., rel.

misc., rel.

Pulkovo. Astronomicheskaia observatorila. Izvestila 21. \#162, 159-161 (1958) (trans1.).

Akademi ia Nauk S.S.S.R. Krymskaia astrofizecheskaia observatoriia. Izvestila 22, 234-256 (1960)

(transl.).

z. Astrophys. 51, 231-285 (1961).

$* * * * *$

Se II

Akademila Nauk S.S.S.R. Krymskaia astrofizecheskaia observatoriia. Izvestila 22, 234-256 (1960).

(transl.).

$* * * * *$

si 1

Astrophys. J. 78, 239-297 (1927).

Monthly Notices Roy. Astron. Soc. 117, 622-628 (1957).

Monthly Notices Roy. Astron. Soc. 117 36-49 (1957) (see also above).

Thes is Harvard (1958).

Spectrochim. Acta 15, 349-359 (1959).

Z. Physik $157,79-\overline{88}(1959)$.

Akademi ia Nauk S.S.S.R. Krymskaia astrofizecheskaia observatoriia. Izvestila 22, 234-256 (1960)

(transl.).

Astrophys.J.Suppl.Ser.5, \#45, 1-137 (1960).

ASTIA Document 239281 (1960).

emiss., rel.

comm。

misc。

comm.

estim.

comm.

emiss.

quant.

emiss.

emiss.

comm.

$\mathrm{CA}$

absorpt.

\section{Si 11}

Astrophys. J. 78, 239-297 (1927).

Nachr. Akad. Wiss. Goottingen Math.-physik. Kl.

$116-118(1946-1948)$. [See al so Biermann, L.

\& Luibeck, K. Z. Astrophys. 25, 325-339 (1948).]

Astrophys. J. 107, 383-385 (1948).

Z. Astrophys. 25, 325-339 (1948).

Publications of the Observatory, Univo of Michigan (Feb. 19, 1954). estim.

SCF

SCF

SCF

misco, rel. 
Varsavsky, C.M.

Hey, P.

Boyarchuk, M. E. E. Boyarchuk, A. A.

Jugaku, Jo, Sargent, L. W. E. Greenstein, J.L.

Russell, H. N.

Aller, L. H. E Jugaku, J.

Gaustad, J.E. E Spitzer, Jr., L.

Russell, H. N.

Rudkjobing, $M$.

Traving, G.

Gaustad, J. E. E Spitzer, Jr., L.

Houziaux, L. E Sadoine, M. P. Douglas, A. S。 E Garstang, R. H

Garstang, R. H.

Allen, C. W.

Allen, C. W. E Asaad, A. S.

Prokof'ev, V. K., Nagibina, 1. M. E Petrova, G. P.

Kerschbaum, $\mathrm{H}$.

Prokof'ev, V. K.

Prokof'ev, V.K.

Kast, W.

Chamalaun, F.J.

King, G. W. E van Vleck, J.H.

Schuttevaer, J.W.

Schuttevaer, J. Wo, de Bont,

M. J. E van den Broek, Th. H.
Thesis Harvard (1958)

Z. Physik 157, 79-88 (1959).

Akademi ia Nauk S.S.S.R. Krymskaia astrofizecheskaia observatorila. Izvestila 22, $234-256$ (1960) (transl.).

Astrophys. J. 134, 783-796 (1961).

quant. emiss. comm.

CA

\section{Si 111}

Astrophys. J. 78, 239-297 (1927).

Astrophys. J. Suppl. Ser. 4, \#38, 109-156 (1959).

Astrophys. J. 134, 771-776 (1961).

estim.

CA

comm. E estim.

\section{Si IV}

Astrophys. J. 78, 239-297 (1927).

Publications of the Copenhagen Observatory No. 145 , $74-82$ (1947).

2. Astrophys. 36, 1-41 (1955).

Astrophys. J. 134, 771-776 (1961).

Bull. soc. roy, sci. Liège 30, 287-299 (1961).

Submitted for publication, Proc. Cambridge Phil. Soc. (1962).

si $x$

Submitted for publication, Ann. astrophys. (1962).

$$
* * * * *
$$

Sn 1

Monthly Notices Roy. Astron. Soc, 117, 622-628 (1957).

Monthly Notices Roy. Astron. Soc. 117, 36-49 (1957) (see al so above).

Optics \& Spectroscopy (U.S.S.R.) 8, 195-197 (1960).

$* * * * *$

Sr 1

Ann. Physik 83, 287-295 (1927).

Z. Physik 50, 70l-715 (1928) (same as below).

Zhur. Ekspti. i Teoret. Fiz, 1, 111-122 (193i)

(transl.) (same as above).

z. Physik 79, 731-735 (1932)

Thesis Utrecht (1934).

Phys. Rev. 56, 464-465 (1939).

Thesis Utrecht (1942) (see below for results). Physica 10, 544-552 (1943). estim. quant.

CA comm.

CA

SCF

quant. forb. comm.

emiss.

emiss. canal

hook, rel. hook, rel.

emiss., rel. emiss., rel.

\& quant. rel.

quant. forb., rel.

emiss., rel. emiss., rel. 
Kruithof, A. M. E Smit, J.A. Eberhagen, A.

Mannkopff, R.

Mitrofanova, L. A.

Ostrovskii, Yu. I., Penkin, N.P. E Shabanova, L. N.

Ostrovskii, Yu. I., Penkin, N. P. E Shabanova, L. N.

Addink, N. W. H.

Dstrovskii, Yu. I. E Penkin, N. P.

Kruithof, A. M. E Smit, J.A. Nikonova, E. I. E Prokof 'ev, V. K.

Dvornikova, I. U. E Nagibina, I. M.

Mi trofanova, L. A.

Ostrovskii, Yu. I. E Penkin, N. P.

Ostrovskii, Yu. I. E Penkin, N.P.

Frerichs, R.

Harrison, G. R.

Harrison, G. R. E Engwicht, $H$.

King, R. B. E King, A. S。

van Stekelenburg, L. H. M.

Petrie, Wo

Rohrlich, F.

van Stekelenburg, L. H. M.

E Smit, J.A.

Mitrofanova, L. A.

Ostrovskii, Yu. I., Parchevskii, G. F. E Penkin, N.P.

Addink, N. W. H.

Allen, C. W.

Boyarchuk, M. E. E Boyarchuk, A. A.

Hefferlin, R., Cobb, B., Hall, D. E Lehman, $C$ 。

Tatum, J.B.
Physica $11,129-143(1944)$ (trans1.).

Z. Physik 143, 392-411 (1955).

Experim. Techn. d. Physik. Sonderheft Spektroskopie $44=50$ (1955).

Pulkovo. Astronomi cheskaia observatori ia. Izvestila 20, \#159, 52-54 (1958) (trans1.).

Bull. Acad. Sci. U.S.S.R. Phys. Ser. 22, 720-724

(1958) (results same as below). [ see also Ostrovskil, Yu. I. EPenkin, N.P., Optics E Spectroscopy (U.S.S.R.) 11, 307-309 (1961).]

Soviet Phys. - Doklady 3, 538-540 (1958) (results same as above). [See also Ostrovskii, Yu. 1. E Penkin, N. P., Optics \& Spectroscopy (U.S.S.R.) $11,307-309$ (1961).]

Spectrochim. Acta 15, 349-359 (1959).

Optics and Spectroscopy (U.S.S.R.) 11, 307-309 (1961).

\section{$\operatorname{Sr} \|$}

Physica 11, 129-143 (1944) (transi.). Optika i Spektroskopiya I, 290-297 (1956) (transl.).

Optika i Spektroskopiya 4, 42I-429 (1958).

Pulkovo. Astronomi cheskaia observatorila. Izvesti ia 20, \#159, 52-54 (1958) (trans1.).

optics and Spectroscopy (U.S.S.R.) 10, 3-6 (1961) (see allso below).

optics and Spectroscopy (U.S.S.R.) 11, 307-309 (1961).

$$
* * * * *
$$

Ti 1

Ann. Physik 81, 807-845 (1926).

J. Opt. Soc. Am. 17, 389-416 (1928).

J. Opt. Soc. Am. 18, 287-301 (1929).

Astrophys. J. 87, 24-39 (1938).

Thesis Utrecht (1943). [See also van Stekelenburg, L. H. M., et al., Physica 14, 189-196 (1948) for results.]

Can. J. Research A 25, $42=48$ (1947).

Phys. Rev。 74, 1381-1396 (1948).

Physica 14, 189-196 (1948).

Pulkovo. Astronomicheskaia observatorila. Izvesti ia 19, \#153, 107-111 (1955) (transl.).

optika i Spektroskopiya 1, 821-832 (1956) (transl.).

Spectrochim. Acta 15, 349-359 (1959).

Monthly Notices Roy. Astron. Soc. 121, 299-332 (1960).

Akademila Nauk S.S.S.R. Krymskaia astrofizecheskaia observatoriia. Izvestila 22, 234-256 (1960) (trans1.).

Astrophys. J. 132, 259-263 (1960).

Monthly Notices Roy. Astron. Soc. $122,311-324$ (1961). estim. emiss. comm.

emiss., rel.

hook

hook

emiss.

hook \& comm.

estim.

hook, rel.

incompl.

emiss., rel.

hook

comm.

emiss., rel. emiss., rel. emiss., rel. absorpt., rel. emiss., rel.

comm.

quant. emiss., rel.

emiss., rel.

hook, rel.

emiss.

comm. \& CA

comm.

emiss., rel.

emiss., rel. 
$\mathrm{Ti} \|$

Harrison, G. R.

King, R. B.

van Stekelenburg, L. H. M.

Petrie, W.

Wilson, 0 . C.

van Stekelenburg, L. H. M. E Smit, J.A.

Aller, L. H., Hazen, M., Doherty, L., Grant, G., Jugaku, J., Spiegel, E. \& Waddell, J.

Mi trofanova, L. A.

Varsavsky, C. M.

Boyarchuk, M. E. E Boyarchuk, A. A.

Rountree, J. C.

Houziaux, L. E Sadoine, M. P. Tatum, J.B.

Kuhn, W。

Kuhn, W。

Prokof'ev, V. K. E Solov'ev, V. N.

Filippov, A. N. E Prokof'ev, V. K.

Muill ler, $F$.

Müll ler, $F$.

Müll ler, $F$.

Prokof'ev, V. K. E Filippov, A. N.

Kvater, G. S.

Kvater, G. S.

Kvater, G. S.

Kvater, G. S.

Kvater, G. S.

Kvater, G. S.

Kvater, G. S.

Stephenson, G. Stephenson, G.

Marr, G. U.
J. Opt. Soc. Am. 17, 389-416 (1928).

Astrophys. J. 94, 27-29 (1941).

Thesis Utrecht (1943). [See also van Stekelenburg,

L. H. M. E Smit, J. A., Physica 14, 189-196 (1948) for results.]

Can. J. Research A 25, 42-48 (1947).

Astrophys. J. 107, 126-150 (1948).

Physica 14, 189-196 (1948).

Publications of the Observatory, Univ. of Michigan (Feb. 19, 1954).

Pulkovo. Astronomicheskaia observatori ia. Izvesti ia 19. \#153, 107-111 (1955) (transl.).

Thesis Harvard (1958).

Akademila Nauk S.S.S.R. Krymskaia astrofizecheskaia observatorila. Izvesti ia 22, 234=256 (1960) (transl.).

Ann. astrophys. 23, 633-654 (1960) (transl.).

Bull. soc. roy. sci. Liège 30, 287-299 (1961).

Monthly Notices Roy. Astron. Soc. 122, $311-324$ (1961).

$$
* * * * * \dot{*}
$$

\section{Tl I}

Naturwissenschaften $13,724-726$ (1925) (see also below).

Kgl. Danske Videnskab. Sel skab Mat.-fys. Medd. Z, misc. $1=86(1926)$.

Z. Physik 48, 276-285 (1928).

Z. Physik 85, 647-660 (1933). [Same as Zhur. Ekspt1. i Teoret. Fiz. 4, 31-42 (1934).]

Helv. Phys. Acta Z, 488-491 (1934).

Helv. Phys. Acta 7, 813-840 (1934).

Helv. Phys. Acta $\frac{7}{8}, 152-164$ (1935).

Zhur. Ekspt1. i Teoret. Fiz. 4, 31-42 (1934) (transl.). [Same as Z. Physik 85, 647-660 (1933).]

Physik. Z. Sowjetunion 7, 226 (1935) (same as below).

Zhur. Eksptl. i Teoret. Fiz. 5, 426-439 (1935) (transl.) (same as above).

Zhur. Eksptl。i Teoret. Fiz, 11, 421-439 (1941). [Same as J. Phys. U.S.S.R. 6, 145-162 (1942); see also Vainshtein, L. A., Bull. Acad. Sci. U.S.S.R. Phys. Ser. 22, 668-699 (1958).]

Zhur. Eksptl.i Teoret. Fiz, 11, 440-447 (1941). [Same as J. Phys. U.S.S.R. 6, 210=217 (1942).]

J. Phys. U.S.S.R. 6, 145-162 (1942). [Same as Zhur. Eksptl. i Teoret. Fiz. 11, $421-439$ (1941).]

J. Phys. U.S.S.R. 6, 210-217 (1942). [Same as. Zhur. Eksptl. i Teoret. Fiz. 11, 440-447 (1941).]

Vestnik Leningrad Univ. Ser. Fiz. i Khim. No.2, $135-141$ (1947) (trans1.).

Nature 167, 112 (1951) (see al so below).

Proc. Phys. Soc. London A 64, 458-464 (1951).

Proc. Roy. Soc. London A 224, 83-90 (1954). emiss., rel. absorpt., rel. emiss., rel.

comm

misco, rel. emiss., rel.

misc., rel.

emiss., rel.

quant.

comm.

comm.

CA

emiss., rel.

misc.

hook, rel.

hook

absorpt. absorpt. absorpt. hook, rel.

hook, rel.

hook, rel.

hook

comm.

hook

comm.

incompl.

misc.

$\mathrm{misc}$.

absorpt. 
Nikonova, E. 1. E Prokof'ev, V. K.

Hinnov, E. E Kohn, H.

Gurvich, L. V.

Vainshtein, L. A.

Brehm, B., Demtröder, W. $\varepsilon$ Osberghaus, 0 .

Demtro̊der, $W$.

Hanus, W.

Tolans, E. L. E Lurio, A.

Demtro̊der, $W$.

Frerichs, R.

King, R. B.

Righini, G.

Ostrovskii, Yu. I.EPenkin, N. P.

Addi nk, N. W. H.

Allen, C. W.

Boyarchuk, M. E. E Boyarchuk, A. A.

Wilson, 0. C.

Aller, L. Ho, Hazen, M., Doherty, L., Grant, G., Jugaku, Jo, spiegel, E. E Waddell, J.

Boyarchuk, M. E. E Boyarchuk, A. A.

Groth, H. G.

Petersen, R.

Edlén, B.

Edlén, B.

Osterbrock, D. E.
Optika i Spektroskopiya 1, 290-297 (1956) (transl.). hook, rel.

J. Opt. Soc. Am, 47, 156-162 (1957). emiss.

Optika i Spektroskopiya 5, 205-207 (1958) (transl.). incompl.

Bull. Acad. Sci. U.S.S.R. Phys. Ser. 22, 668-669 (1958).

Z. Naturforsch. 16a, 843 (1961). [See al so Demtro̊der, W., below and $Z_{\text {. Physik } 166,42-45}$ (1961).]

Thesis Bonn (1961). [See Z. Physik 166, 42-45 (1962) for results.]

Bull. acad. polon. sci. Classe 3, 8, 629-636 (1961). quant.

Bull. Am. Phys. Soc. (2) $6,75(1961)$ misc。

Z. Physik 166, 42-45 (1962).

life

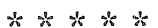

V I

Ann. Physik 81, 807-845 (1926).

Astrophys. J. 105, 376-389 (1947).

Mem. soc. astron. ital . 19-20, 303-311 (ed.

Allen, C. W., University Press, Princeton, 1948-1949) (trans1.).

Optika i Spektroskopiya 5, 345-353 (1958) (transl.). hook, rel.

Spectrochim. Acta 15, 349-359 (1959).

Monthly Notices Roy. Astron. Soc. 121, 299=332 (1960).

Akademi ia Nauk S. S.S.R. Krymskaia astrofizecheskaia observatorila. Izvestila 22, 234-256 (1960) (transl.).

\section{V \|}

Astrophys. J. 107, 126=150 (1948).

Publications of the Observatory, Univ. of Michigan (Feb. 19, 1954).

Akademi ia Nauk S.S.S.R. Krymskaia astrofizecheskaia observatorila. Izvestila 22, 234-256 (1960) (trans1.).

z. Astrophys. 51, 231-285 (1961).

emiss., rel. absorpt., rel. misc., rel.

emiss. comm。 \& CA

comm。

misc., rel. misc., rel.

comm。

misc.

$$
* * * * *
$$

Xe 1

Phys. E Chem. Solids 1, 284 (1957).

quant., rel.

$\mathrm{Xe} \|$

Phys. Rev. 65, 248 (1948).

quant. forb.

$\mathrm{Xe} \| \mathrm{H}$

Phys。 Rev。 65, 248 (1948)。

Astrophys. J. 114, 469-472 (1951).

quant. forb. quant. forb. 
Y I

Goldberg, L., Mi̊ller, E. A. \& Aller, L. H.

Boyarchuk, M. E. E Boyarchuk, A. A.

Russel 1, H. N.

Prokof'ev, V. K.

Larché, K.

Prokof'ev, V. K.

Filippov, A. N.

Filippov, A. N.

Soleillet, P.

Billeter, $W$.

Billeter, W.

Soleillet, P.

Auslånder, J.

Mason, R. C.

Bruck, $H$.

King, G. W. E van Vleck, J. H.

Bruck, $H$.

Bruck, H.

Schuttevaer, J.W.

Schuttevaer, J. W. E Smit, J. A.

Schuttevaer, J. W., de Bont,

M. J. E van der Broek, Th. H.

Spitzer, M.

Geneux, E. E Wanders-Vincenz, B.

May, A. D.

May, A. D.

Penkin, N. P. E Red'ko, T. P.
Astrophys. J. Suppl. Ser. 5, $\# 45,1-137$ (1960).

$Y \|$

Akademila Nauk S.S.S.R. Krymskaia astrofizecheskaia observatorila. Izvestila 22, 234-256 (1960)

(transl.).

$$
* * * * *
$$

$\mathrm{Zn} \mathrm{I}$

Astrophys. J. 78, 239-297 (1927).

Z. Physik 50,701-715 (1928). [Same as Zhur.

Ekspt1. i Teoret. Fiz. 1, $111-122$ (1931).]

Z. Physik 67, 440-477 (1931).

Zhur. Eksptl. i Teoret. Fiz. 1, 111-122 (1931)

(transl.). [Same as Z. Physik 50, $701-715$ (1928).]

Physik. Z. Sowjetunion 1, 289-296 (1932) (same as below).

Trudy Gosudarst. Opt. Inst. Leningrad $8,1-8$ (1932) (transl.) (same as above).

Compt. rend. 195, 372-373 (1932) (transl.).

Helv. Phys. Acta 7, 505-513 (1934).

Helv. Phys. Acta Z, 841-842 (1934).

Compt. rend. 204, 253-255 (1937).

Helv. Phys. Acta 11, 562-586 (1938).

Physica 5, 777-783 (1938).

Compt. rend. 208, 1805-1807 (1939) (transi.).

Phys. Rev. 56, $464-465$ (1939).

Thesis Paris (1942) (trans1.).

Compt. rend. 214, 307-309 (1942) (transl.).

Thesis Utrecht (1942). [See al so below and

Schuttevaer, J.W., et al., Physica 10, 544-552 (1943).]

Physica 10, 502-512 (1943) (see al so below).

Physica 10, 544-552 (1943).

Compt. rend. 239, 696-698 (1954) (transl.).

Helv. Phys. Acta 33, 185-220 (1960) (transl.).

Compt. rend. 250, 3616-3617 (1960) (transl.).

Compt. rend. 251, 1371-1372 (1960) (transi.).

Optics and Spectroscopy (U.S.S.R.) 2, 360 (1960).
CA

comm., rel. estim.

estim.

misc., rel. estim.

hook, rel.

hook, rel.

misc.

absorpt.

comm.

absorpt.

absorpt.

emiss., rel.

misc.

quant. forb., rel.

misc.

misc.

emiss., rel.

emiss., rel.

emiss., rel.

misc.

misc.

misc.

misc.

hook, rel.

$\varepsilon$ emiss., rel.
Russell, H. N.

Bruck, $H$.

Geneux, E. \& Wanders-Vincenz, B.
Astrophys. J. 78, 239-297 (1927).

Compt. rend. 208, 1805-1807 (1939) (transl.).

Helv. Phys. Acta $33,185-220$ (1960) (transl.). estim.

misc.

misc。 


\section{$\operatorname{Zr}$}

Goldberg, L., Milller, E. A. \& Aller, L. H.

\section{Zr II}

Boyarchuk, M. E. E Boyarchuk, A. A.

Houziaux, L. E Sadoine, M. P. (trans1.).
Astrophys. J. Suppl. Ser. 5, t45, 1-137 (1960).

CA

Akademila Nauk S.S.S.R. Krymskaia astrofizecheskaia observatoriia. Izvesti ia 22, 234-256 (1960)

Bull. soc, roy, sci. Liège $30,287-299$ (1961).

$* * * * *$ comm., rel.

CA 


\section{PARTIAL LIST OF ABBREVIATIONS FOR JOURNALS}

For convenience complete titles for the abbreviations of sparsely circulated journals are listed.

$A E C=T R$

Ann. astrophys.

Arch. néerl. sci. $3 A$

ASTIA

Astron. Zhur.

Soviet Astronomy-AJ

ATS (transl.)

Bull. acad. polon. sci. Classe 3

Bull. soc. roy. sci. Liège

Compt. rend.

Doklady Akad. Nauk Armyan. S.S.R.

Doklady Akad. Nauk S.S.S.R.

Soviet Phys.-Doklady

Ergeb. exakt. Naturw.

Geofys. Publikasjoner Norske Videnskaps-Akad. i Oslo

Izvest. Akad. Nauk S.S.S.R. Ser. Fiz.

Bull. Acad. Sci. U.S.S.R. Phys. Ser.

J. Atmospheric and Terrest. Phys.

J. phys. radium

J. Phys. (U.S.S.R.)

J. Tenn. Acad. Sci.

Kgl. Danske Videnskab. Selskab Mat.-fys. Medd.

Kgl. Norske Videnskab. Selskabs, Skrifter

Mem. soc. astron. ital.

Mém. soc. roy. sci. Liège

Muinch. tierårztl. Wochschr.

Nachr. Akad. Wiss. Go̊ttingen, Math.-physik. KI.

Optika i Spektroskopiya

Optics and Spectroscopy (U.S.S.R.)

sitzber. deut. Akad. Wiss. Berlin, Kl. Math., Phys. u Tech.

Si tzber. math.-physik. Kl . bayer. Akad. Wiss. München

SLA-RT

Trudy Gosudarst. Opt. Inst. Leningrad

Uspekhi Fiz. Nauk

Verhandl. deut. physik. Ges.

Z. El ektrochem。

Z. physik. Chem.

Zhur. Eksptl.i Teoret. Fiz.

Soviet Phys - JETP
Atomic Energy Commission English translation

Annales d'astrophysique

Archives néerlandaises des sciences exactes et naturelles. Series $3 A$

Armed Services Technical Information Agency

Astronomi cheski i Zhurnal

Astronomical Journal (English translation of above)

English translation available from:

Associated Technical Services, Inc.

P. 0. Box 271, East Orange, New Jersey

Bulletin de l'académie polonaise des sciences. Classe 3

Bulletin de la société royale des sciences de Liège

Academie des sciences, Paris. Comptes rendus

Doklady Akademi i Nauk Armyanskoi. S.S.R.

Doklady Akademi i Nauk S. S.S.R.

Proceedings of the Academy of Sciences of the U.S.S.R. (English translation of above)

Ergebnisse der exakten Naturwi ssenschaften Geofysiske Publikasjoner. Utgitt av det Norske Videnskaps-Akademi i Oslo

Izvestiya Akademi i Nauk S.S.S.R. Seriya Fizicheskaya Bulletin of the Academy of Science of the U.S.S.R. Physical Series (English translation of above)

Journal of Atmospheric and Terrestial Physics

Journal de physique, Le, et Le radium

Journal of Physics (Academy of Sciences of the U.S.S.R.) (in English)

Journal of the Tennessee Academy of Science Kongelige Danske Videnskabernes Selskab, Det, Mathematisk-fysiske Meddelelser

Kongelige Norske Videnskabers Selskabs, Det, Skrifter

Memorie della societá astronomica italiana

Mémoires de la société royale des sciences de Liège

Münchener tierårztliche Wochenschrift

Nachrichten der Akademie der Wissenschaften in Go̊ttingen, Mathematisch-physikalische Klasse (no abbreviation) (no abbreviation) (English translation of above)

Sitzungsberichte der deutschen Akademie der Wissenschaften zu Berlin, Klasse für Mathematik, Physik und Technik

Sitzungsberichte der mathematisch-physikal ischen Klasse der bayerischen Akademie der Wissenschaften zu München

English translation available from:

SLA Translation Monthly, The John Crerar Library, 86 East Randolph St., Chicago, 111 inois

Trudy Gosudarstvennogo Opticheskogo Instituta, Leningrad

Uspekhi Fizicheskikh Nauk

Verhandlungen der deutschen physikalischen Gesell lschaft

Zeitschrift für Elektrochemie

Zeitschrift für physikalische Chemie (Leipzig)

Zhurnal Eksperimental "noi i Teoreticheskoi Fiziki Journal of Experimental and Theoretical Physics (English translation of above) 


\section{Selected Publications of the National Bureau of Standards}

Atomic Energy Levels, C. E. Moore:

Circular 467, Volume I. $H$ to $\mathbb{V}(\mathbb{Z}=1$ to 23) 206 spectra. 309 p. (1949)

$\$ 5.50$

Circular 467, Volume II. Cr to $\mathrm{Nb}(\mathbb{Z}=24$ to 41$) 152$ spectra. 227 p. (1952)

Circular 467 , Volume III. $\left\{\begin{array}{l}\left.\text { Mo to } \mathrm{La}_{(\mathbb{Z}}=42 \text { to } 57\right) \\ \mathrm{H} f \text { to } \mathrm{Ac}(\mathbb{Z}=72 \text { to } 89)\end{array}\right\} 124$ spectra. 245 p. (1958)

$\$ 4.00$

$\$ 2.50$

An Ultraviolet Multiplet Table, C. $\mathbb{E}$. Moore:

Circular 488, Section 1. $H$ to $V(Z=1$ to 23$)$; Selected Multiplets of 79 Spectra. 78 p. (1950).

Circular 488 , Section 2. $\mathrm{Cr}$ to $\mathrm{Nb}$ ( $\mathrm{Z}=24$ to 41 ); Selected Multiplets of 46 Spectra. 115 p. (1952).

Circular 488, Section 3. $\left\{\begin{array}{l}\text { Mo to } \mathrm{La}(\mathbb{Z}=42 \text { to } 57) \\ \text { Hf to } \mathbb{R a}(Z=72 \text { to } 88)\end{array}\right\}$ Selected Multiplets of 78

$\$ 0.70$

Spectra. 94 p. (1961).

Circular 488 , Section 4 . $\mathbb{H}$ to $\mathrm{Nb}(\mathrm{Z}=1$ to 41$)$; Finding List for Sections 1 and 2 of the Table. 65 p. (1961).

Circular 488, Section 5. $\quad\left\{\begin{array}{l}\text { Mo to } \mathrm{La}(Z=42 \text { to } 57) \\ \mathrm{Hf} \text { to } \mathrm{Ra}(\mathrm{Z}=72 \text { to } 88)\end{array}\right\}$ Finding List for Section 3 of the Table. 30 p. (1961).

$\$ 0.60$

$\$ 0.45$

$\$ 0.30$

Table of Wavenumbers, C. D. Coleman, W. R. Bozman, and W. F. Meggers:

Monograph 3, Volume I. 2000 A to 7000 A. 508 p. (1960)

Monograph 3, Volume II. 7000 A to $1000 \mu$. 542 p. (1960)

$\$ 6.00$

$\$ 6.00$

New Description of Thorium Spectra, Romuald Zalubas:

Monograph 17, 106 p. (1960)

\$0. 65

Tables of Spectral-Line Intensities, W. F. Meggers, C. H. Corliss, and B. F. Scribner:

Monograph 32, Part I. Arranged by Elements. 474 p. (1961) $\$ 400$

Monograph 32, Part II. Arranged by Wavelengths. 272 p. (1961) \$3.00

The above publications may be purchased from the Superintendent of Documents, U.S. Government Printing Office, Washington 25, D.C.

A Multiplet Table of Astrophysical Interest, C. E. Moore. A Reprinting of the 1945 Multiplet Table (Princeton Univ., Obs. Contr. No. 20):

Technical Note 36 (PB151395), Part I Table of Multiplets, and Part II Finding List, $\lambda \lambda 2951 \AA-13164 \AA$. 242 p. (1959).

The above Technical Note may be purchased by the PB number from the Department of Commerce, Office of Technical Services, Washington 25, D.C. 



U.S. Department of Commerce,

Luther H. Hodges, Secretary.

National Bureau of Standards,

A. V. Astin, Director.

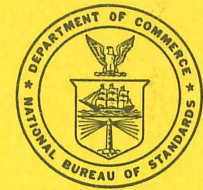

\section{THE NATIONAL BUREAU OF STANDARDS}

The scope of activities of the National Bureau of Standards at its major laboratories in Washington, D.C., and Boulder, Colorado, is suggested in the following listing of the divisions and sections engaged in technical work. In general, each section carries out specialized research, development, and engineering in the field indicated by its title. A brief description of the activities, and of the resultant publications, appears on the inside of the front cover.

WASHINGTON, D.C.

Electricity. Resistance and Reactance. Electrochemistry. Electrical Instruments. Magnetic Measurements. Dielectrics. High Voltage.

Metrology. Photometry and Colorimetry. Refractometry. Photographic Research. Length. Engineering Metrology. Mass and Scale. Volumetry and Densimetry.

Heat. Temperature Physics. Heat Measurements. Cryogenic Physics. Equation of State. Statistical Physics. Radiation Physics. X-ray. Radioactivity. Radiation Theory. High Energy Radiation. Radiological Equipment. Nucleonic Instrumentation. Neutron Physics.

Analytical and Inorganic Chemistry. Pure Substances. Spectrochemistry. Solution Chemistry. Standard Reference Materials. Applied Analytical Research. Crystal Chemistry.

Mechanics. Sound. Pressure and Vacuum. Fluild Mechanics. Engineering Mechanics. Rheology. Combustion Controls.

Polymers. Macromolecules: Synthesis and Structure. Polymer Chemistry. Polymer Physics. Polymer Characterization. Polymer Evaluation and Testing. Applied Polymer Standards and Research. Dental Research.

Metallurgy. Engineering Metallurgy. Microscopy and Diffraction. Metal Reactions. Metal Physics. Electrolysis and Metal Deposition.

Inorganic Solids. Engineering Ceramics. Glass. Solid State Chemistry. Crystal Growth. Physical Properties. Crystallography.

Building Research. Structural Engineering. Fire Research. Mechanical Systems. Organic Building Materials. Codes and Safety Standards. Heat Transfer. Inorganic Building Materials. Metallic Building Materials.

Applied Mathematics. Numerical Analysis. Computation. Statistical Engineering. Mathematical Physics. Operations Research.

Data Processing Systems. Components and Techniques. Computer Technology. Measurements Automation. Engineering Applications. Systems Analysis.

Atomic Physics. Spectroscopy. Infrared Spectroscopy. Solid State Physics. Electron Physics. Atomic Physics. Instrumentation. Engineering Electronics. Electron Devices. Electronic Instrumentation. Mechanical Instruments. Basic Instrumentation.

Physical Chemistry. Thermochemistry. Surface Chemistry. Organic Chemistry. Molecular Spectroscopy. Molecular Kinetics. Mass Spectrometry.

Office of Weights and Measures.

BOULDER, COLO.

Cryogenic Engineering Laboratory. Cryogenic Equipment. Cryogenic Processes. Properties of Materials. Cryogenic Technical Services.

\section{CENTRAL RADIO PROPAGATION LABORATORY}

Ionosphere Research and Propagation. Low Frequency and Very Low Frequency Research. Ionosphere Research. Prediction Services. Sun-Earth Relationships. Field Engineering. Radio Warning Services. Vertical Soundings Research.

Radio Propagation Engineering. Data Reduction Instrumentation. Radio Noise. Tropospheric Measurements. Tropospheric Analysis. Propagation-Terrain Effects. Radio-Meteorology. Lower Atmosphere Physics.

Radio Systems. Applied Electromagnetic Theory. High Frequency and Very High Frequency Research. Modulation Research. Antenna Research. Navigation Systems.

Upper Atmosphere and Space Physics. Upper Atmosphere and Plasma Physics. Ionosphere and Exosphere Scatter. Airglow and Aurora. Ionospheric Radio Astronomy.

\section{RADIO STANDARDS LABORATORY}

Radio Physics. Radio Broadcast Service. Radio and Microwave Materials. Atomic Frequency and Time-Interval Standards. Millimeter-Wave Research.

Circuit Standards. High Frequency Electrical Standards. Microwave Circuit Standards. Electronic Calibration Center. 
